\title{
Secondary sulphate minerals from Bhanine Valley coals (South Lebanon) - a crystallochemical and geochemical study
}

\author{
Łukasz KRUSZEWSKI ${ }^{1, *}$ \\ 1 Polish Academy of Sciences, Institute of Geological Sciences, Twarda 51/55, 00-818 Warsaw, Poland
}

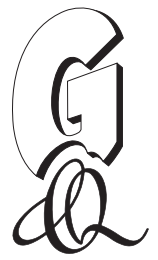

Kruszewski, Ł., 2019. Secondary sulphate minerals from Bhanine Valley coals (South Lebanon) - a crystallochemical and geochemical study. Geological Quarterly, 63 (1): 65-87, doi: 10.7306/gq.1450

Associate editor: Tomasz Bajda

Rich efflorescences of various Fe and Al sulphate mineral mixtures on coal seams of Bhanine, South Lebanon, were examined using (1) Powder X-Ray Diffraction (with the Rietveld method and unit cell parameters calculation), (2) Scanning Electron Microscopy with standardized Electron-Dispersive Spectroscopy system, and (3) Inductively-Coupled Plasma Mass Spectroscopy. The sulphates most likely originated from coal-contained pyrite to form Fe(II) sulphates (melanterite, rozenite, and the most common szomolnokite), followed by $\mathrm{Fe}^{3+}$-rich sulphates (coquimbite group, copiapite group) and $\mathrm{Al}$ sulphates (alunogen, tamarugite). The halotrichite group and minor voltaite, metavoltine, and possibly secondary rozenite and szomolnokite were the last species to be formed. Strong enrichment in Al in copiapites and coquimbites, common occurrence of aluminocoquimbite, and Al likely entering the structure of $\mathrm{Fe}(\mathrm{II})$ sulphates makefurther phenomena, during which the initial ferrous copiapites were oxidized in the presence of Al-rich solutions, not out of the question. The obtained unit cell parameters sometimes stand for threshold values in the literature-based ranges drawn, but the values are usually below the $2 \%$ discrepancy. The Bhanine sulphates bear relatively large amounts of $\mathrm{TI}, \mathrm{Hg}$, and Co when compared to Coal Clarke and mean crustal abundancies, being also moderately enriched in $\mathrm{Ni}$ and $\mathrm{As}$.

Key words: iron sulphates, aluminium sulphates, coquimbite, coal, unit cell parameters, standardized Energy Dispersive Spectroscopic analysis.

\section{INTRODUCTION}

Iron and aluminium sulphates (IAS) are common secondary minerals formed in expense of reduced-sulphur compounds, like ore minerals (sulphides, sulphosalts) and organic sulphur compounds in coal (e.g., Joeckel et al., 2005). An important source of such sulphates is a thoroughly studied phenomenon known as acid mine drainage (e.g., Keith et al., 2001; Hammarstrom et al., 2005; Romero et al., 2006; Bobos et al., 2006; Triantafyllidis and Skarpelis, 2006; Smuda et al., 2007; Welch et al., 2009; Fitzpatrick et al., 2010). Other studies related to such sulphate occurrences in the vicinities of ore deposits include those of Cooper et al. (2008), while those from volcanic zones were examined by, e.g. Martin et al. (1999), Rodgers et al. (2000) and Zimbelman et al. (2005). A separate, but at least the same important topic related to secondary sulphate minerals (mainly Fe- and Mg-rich ones) is their either confirmed or suggested occurrence at the surface of Mars (e.g., Gornitz, 2004, 2005; Chipera, Vaniman, 2007; Sgavetti et al.,

\footnotetext{
*E-mail: Ikruszewski@twarda.pan.pl

Received: August 23, 2018; accepted: November 25, 2018; first published online: February 7, 2019
}

2009; Rice et al., 2010). Being associated with the AMD, IAS may immobilize essential amounts of toxic elements (e.g., Co, $\mathrm{Cu}, \mathrm{Zn}, \mathrm{As}, \mathrm{Pb}$; Buckby et al., 2003; Jamieson et al., 2005; Bobos et al., 2006; Majzlan and Michalik, 2007).

Sulphates are commonly found as secondary minerals in coal seams, especially those exposed to atmospheric air. They usually form due to the oxidation of pyrite and other sulphide minerals disseminated in coaly matter (e.g., Gluskoter, 1977; Montano, 1981; Ward, 2002; Kostova et al., 2005; Stracher et al., 2005; Susilawati and Ward, 2006; Murray et al., 2014; Bielowicz, Misiak, 2016; Dai et al., 2017; Liu et al., 2018). Young and Nancarrow (1988) report an occurrence of rozenite-copiapite-pickeringite-epsomite-jarosite-gypsum encrustationson coals from Cumbria, Great Britain. Three different copiapite group minerals, namely aluminocopiapite, copiapite and magnesiocopiapite, were described from a similar geological environment in Nova Scotia, Canada (Zodrow, 1980). A paper by Matýsek et al. (2014) on brine-bearing coal-associated sulphates (including $\mathrm{Na}-\mathrm{Fe}$ sulphate metasideronatrite) from the Czech part of the Upper Silesian Basin is noteworthy. A similar paper by Zielinski et al. (2001) gives data on natrojarosite and $\mathrm{Na}$ - and Mg-rich sulphates in relation to the coal-brine environment of a mine in Colorado, USA. An occurrence of some Fe sulphates in relation to both acid mine drainage and coal is described, e.g. by Wisotzky and Obermann (2001). Also alunite-group sulphates 
are known to occur within coal deposits (e.g., Ward et al., 2002; Lipiarski et al., 2004).

A separate case concerns formation of sulphate species due to coal fires, both natural and anthropogenic (e.g., Blass and Strehler, 1993; Filippidis et al., 1996; Witzke, 1996; Ward, 2002; Stracher et al., 2005; Masalehdani et al., 2009). This formation takes place in the course of two main fire-triggered processes:

- condensation of coal-fire gaseous jets and their pneumatolytic-like and hydrothermal interaction with surrounding rocks by relatively high temperatures even exceeding $500^{\circ} \mathrm{C}$, and/or

- oxidation of primary S species (gaseous and liquid ones) within local supergene zones (Dokoupilová et al., 2007; Kruszewski, 2013).

The latter process resembles ore oxidation taking place in aeration zones of deposits. Sulphate minerals are also found in low-temperature ashes of low-rank coal combustion, standing for their important components (e.g., Ward, 2002, 2016; Dai et al., 2015; Liu et al., 2018).

During the archeological "Eschmoun Valley" mission to the Aouali Valley (also known as the Eschmoun Valley) in south Lebanon, led by the Institute of Archaeology, University of Warsaw, Poland, a colleague (Maciej Krajcarz) conducting geological research found secondary sulphate minerals in a scenery described in detail below. The samples were brought to the author by him for a study, the results of which are presented herein. The goal of this paper is to characterize the chemical nature of these minerals and to juxtapose their crystallographical character with the known, though often scarce, literature data. It is noteworthy that sulphate minerals described below were not known from Lebanon before.

\section{GEOLOGICAL SETTING}

The coal occurrence is located in the vicinity of the city of Bhanine, in the Mohafazat Liban-Sud, Jezzine district (about $5 \mathrm{~km}$ NE from Jezzine, between the towns of Jezzine and Betir El Shouf). This sandpit locality $\left(33^{\circ} 34^{\prime} 58^{\prime \prime} \mathrm{N} 35^{\circ} 35^{\prime} 58^{\prime \prime} \mathrm{E}\right)$ is not far from the confluence of the Aouali (Eshmoun) and Bhanine valleys (Fig. 1).
The sandpit belongs to the Chouf Sandstone Formation occurring in this area. It is the lowest Cretaceous unit in Lebanon and comprises ferruginous, brown to white sandstone accompanied by clays, shales and lignites. Woody and coaly fragments found within the Chouf sandstones contain Fe sulphides (pyrite and marcasite) and amber. Basalts and clayey tuffs are also found within it. The formation is likely of fluvial to deltaic and littoral origin (Walley, 1997). A stream is noticed running from the sandpit, further forming a waterfall. Sandstone samples collected at the waterfall point contain abundant pyrite as a glue material. The sandstone is, however, clearly zoned, and some of its parts are devoid of pyrite but are rusty-brown, suggesting the presence of goethite (or a mixture of iron oxyhydroxides). Although no direct information about the use of the local coal could be found, according to ELARD (2017) coal is found in some waste deposits in the vicinity of Saida. The presence of coal and an iron ore in the Jezzine area is also mentioned by Nanhas (2012). A coal quarry and coal mines in the nearby village of Kaitouly (Qaytoula), located to the south and north of its centre, respectively, are found in a map provided by the Lebanon ministry of tourism (Lebanon..., 2011).

\section{ANALYTICAL METHODS}

The initial Powder X-Ray Diffraction (PXRD) analyses were performed on the Bruker axs D5005 diffractometer, equipped with a graphite monochromator, at the Department of Soil Environment Sciences of the Faculty of Biology and Agriculture, Warsaw Agriculture University (Poland). The measurement parameters were as follows: Co K $\alpha$ radiation (Fe-filtered), 0.02 (20) step, 3s counting time per step. The most important samples were re-analysed using the Bruker axs D8 ADVANCE diffractometer with a super-fast VÅNTEC-1 linear position-sensitive (LPSD) detector; the apparatus was located at the Institute of Geological Sciences, Polish Academy of Sciences, War-

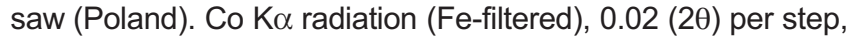
$1 \mathrm{~s}$ counting time (equal to $\sim 416 \mathrm{~s}$ of the zero-dimensional scintillation counter). TOPAS (v. 3) software was used for the unit cell parameter refinements; the refinements were carried out using the Rietveld method with a standard-set full axial model. As the used software version is non-compatible with the LSPD detec-

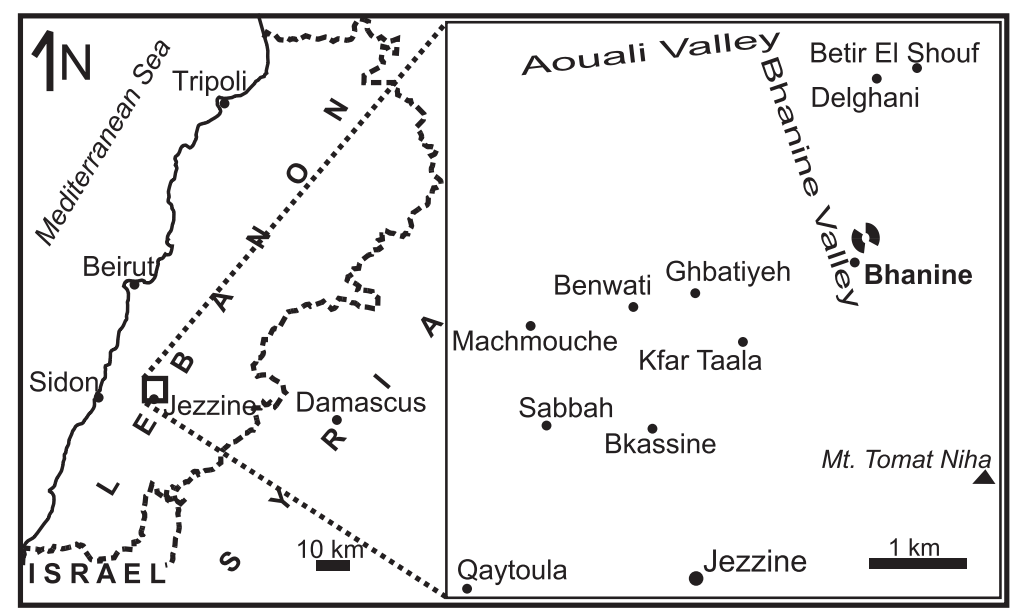

Fig. 1. Location of the coal-sulphate occurrence in South Lebanon 
tor, the influence of thus lacking receiving slit was imitated by additional convolution input. For this purpose, i.e., to obtain the correct instrumental-dependent profile and peak shape, LaB6 standard (NIST SRM 660c) was first analysed, its known unit cell parameter and mean crystal size fixed, and then all the available functions were checked for their influence on refinement. The Lorentzian function with constant $2 \theta$ dependence was found to be the only function being physically meaningful for the profile description. This procedure was applied based on information from David Bish (pers. comm., 2012). Influence of both the crystal size and strain in both Gaussian and Lorentzian dependence was observed and used only when the values obtained were larger than errors. The refinements were run in the mid-angle range due to a bias possible for the high-angle range (e.g., Pecharsky and Zavalij, 2003). Graphic fit was controlled. Various models, using different structure files (i.e. structure models available in the literature), were tried. The results with best $R_{\mathrm{wp}}$ (residual weighted-pattern) and GOF (goodness of fit, $\left.\chi^{2}\right)$ statistics were chosen as the final ones.

Scanning electron microscope (JEOL JSM/JXM 840A apparatus at the Institute of Geological Sciences, Polish Academy of Sciences) with an energy-dispersive spectroscopy detector was used to (1) visualize the habit and spatial dependence of minerals and (2) conduct standardized EDS analyses of minerals. The latter method was successfully used by the author for a similar research before (Kruszewski, 2013), and it was shown to be less destructive than the commonly used wavelength mode and thus more accurate for sulphate minerals analysis. The beam current used was $15 \mathrm{kV}$ and $1 \mathrm{nA}$. Partial sample destruction still present and very strong tendency for the minerals under study to form intergrowths made the mineral analysis difficult. Thus, to obtain the best results, the analytical results were compared to the literature data and ideal compositions (from the Handbook of Mineralogy, Anthony et al., 2003) of the particular mineral species otherwise confirmed via PXRD. Analytical results were recalculated with factors standing for the ideal sum of cations with an exception of the copiapite group, for which the author related the ideal sum of tetrahedral atoms. Addressing cations instead of oxygen allows omitting difficulties with $\mathrm{Fe}^{2+}-\mathrm{Fe}^{3+}$ division and unknown true water content. Some elements (e.g., $\mathrm{Na}, \mathrm{K}, \mathrm{Ca}, \mathrm{Si}$ ), which seem not to fit particular mineral structures, were omitted in the calculation of crystallochemical formulas. Some elements analysed are also hard to be confirmed due to strong line coincidences in the EDS spectra. This has especially to do with the $\mathrm{Sr}-\mathrm{Si}$ and $\mathrm{Na}-\mathrm{Zn}$ pairs. It should also be noted that the water content, although reported, is arbitrary, as it is just calculated by difference. Its content strongly depends on the level of particular crystal destruction under the electron beam. Due to the above considerations, many of the empirical formulas presented below should be treated as an approximation of the particular species crystallochemistry.

Concentrations of trace elements in bulk sulphate samples were analysed using the Inductively CoupledPlasma (ICP) Optical Emission Spectroscopy (OES) method. The apparatus used - Optima D5000 DV of Perkin Elmer - was located at the Laboratory of Water, Soil and Rock Chemistry of the Faculty of Geology, University of Warsaw.

\section{ASSEMBLAGES AND MINERAL HABIT}

As the mineral samples were collected in few areas, many of them coming from profiles, some parageneses can be listed
(Table 1). In the vicinity of a local stream, simple hydrated $\mathrm{Fe}^{2+}$ sulphates (melanterite, rozenite) and gypsum are the dominant species. Samples No. 03 and No. 39 are of particular interest, as their PXRD spectra show quartz only. When analysed via SEM-EDS, no crystals can be seen; the such-viewed accumulations show some Al admixture. It is suggested that these samples represent a partially crystallized silica gel. Phase composition of Profile I is much more complex, with $\mathrm{Fe}^{3+}-\mathrm{Al}, \mathrm{Fe}^{2+}-\mathrm{Fe}^{3+}$ and $\mathrm{Na}-\mathrm{Al}-\mathrm{Fe}^{3+}(\mathrm{Mg})$ systems being represented. Na-Al system is present in Profiles II and V. The possible coexistence of the sulphate minerals in sample Aou21 of the latter Profile with nordstrandite, $\mathrm{Al}(\mathrm{OH})_{3}$, is worth of notice, as this mineral is also reported from some burning coal-mining heaps (Kruszewski et al., 2018a). Profile III is largely aluminous, with the upper part characterized as the $\mathrm{Fe}^{3+}$-Al-Na, and the lower one being devoid of $\mathrm{Na}$ compounds but enriched in $\mathrm{Ca}$. The occurrence of native sulphur admixture in one of the profile's sample (Aou34) is noteworthy, as this mineral is commonly found in fire zones of coal-mining heaps (e.g., Kruszewski et al., 2018a). The chemical characteristics of Profile IV are either of the Fe-Al or rather purely ferric type. The coal-rich areas distinguish themselves in the presence of $\mathrm{Na}-\mathrm{Fe}$ sulphates. Samples that crystallized directly below the coal deposits are devoid of $\mathrm{Na}$ but enriched in Al. Sample Aou31 is interesting due to its possible trace mikasaite (which is anhydrous mineral) and rare $\mathrm{CaCO}_{3}$ modification vaterite (which is relatively frequently observed in burning fossil-fuel waste heaps; e.g., Kruszewski, 2006; Kruszewski et al., $2018 b)$. At least few of the "unknown source" samples are noteworthy due to their potential containment of interesting and possibly fingerprint minerals. The first one is sample Aou30 where boussingaultite may be present; the mineral is $\mathrm{NH}_{4}$-bearing and the ammonium ion is typical for coal-fire zones of some heaps (e.g., Kruszewski, 2013; Kruszewski et al., 2018a). The other samples are listed here due to similar reasons. Theseare Aou17 and Aou36, with possible rostite; and Aou 18, with possible sassolite $\left(\mathrm{H}_{3} \mathrm{BO}_{3}\right)$. Confirmation of the presence of the Cu-bearing mineral chalcoalumite in sample Aou29-2 has failed. So is true for the potentially Cu-bearing aubertite. It is supposed that such minerals have been torn away during the thin-section separation, diminished due to their deliquescence. It should be noticed that voltaite, although evident in some of the samples studied, is present only at their surface. It thus does not stand an important constituent of the assemblages characterized.

The habit of the particular minerals and their aggregates can be seen in some images juxtaposed (Fig. 2). The SEM images of some minerals are present in Figure 3. Figures $2 \mathrm{~A}$ and $3 \mathrm{~A}$ show, respectively, botryoidal aggregates and intergrown crystals of rozenite. The latter form vermicular aggregates. Rozenite crystals are usually up to $12 \mu \mathrm{m}$ in diameter, but the crystal edges can reach $15 \mu \mathrm{m}$. The coquimbite and copiapite group form rather compact aggregates in this particular sample. Better depicted botryoidal aggregates composed of minerals of the copiapite group can be seen in Figure 2C. Individual copiapite group botryoids can reach or slightly exceed $2 \mathrm{~mm}$ in diameter. Although present in Figure 2D in minor amounts, macroscopically good crystals of the coquimbite group could not be found. However, they can be seen in the SEM image (Fig. 3F) as rosette-like aggregates. The aggregates reach $\sim 200 \mu \mathrm{m}$ in diameter, while the largest crystals $-\sim 60 \mu \mathrm{m}$. A triangular section of paracoquimbite crystal, $\sim 20 \mu \mathrm{m}$ in diameter, is also observed in Figure 2D. Needle-like crystals and their aggregates of halotrichite are shown in Figures 2B, D and 3A. Their length is usually up to $100 \mu \mathrm{m}$, but can reach $0.7 \mathrm{~mm}$. Tamarugite and alunogen are usually found as tightly packed crystalline aggre- 
PXRD qualitative phase analysis results for the researched sulphate mineral samples

\begin{tabular}{|c|c|c|}
\hline $\begin{array}{l}\text { Macroscopic description } \\
\text { and location details }\end{array}$ & Confirmed minerals & Questionable trace minerals \\
\hline \multicolumn{3}{|c|}{ Stream vicinity } \\
\hline greenish somewhat fibrous masses & melanterite ${ }^{1}$, rozenite, coquimbite, copiapite group, gypsum & $\begin{array}{l}\text { rhomboclase, starkeyite, } \\
\text { kieserite, cristobalite }\end{array}$ \\
\hline \multirow[t]{2}{*}{ scarce white cryptocrystalline encrustations } & quartz & \\
\hline & gypsum, quartz & $\begin{array}{l}\text { No. 38: baryte, gunningite, } \\
\text { ammonioalunite, darapskite }\end{array}$ \\
\hline greenish aggregates & $\begin{array}{c}\text { rozenite, coquimbite, paracoquimbite, szomolnokite, } \\
\text { copiapite g., quartz }\end{array}$ & \\
\hline \multicolumn{3}{|c|}{ PROFILE I } \\
\hline yellow aggregates & copiapite g., coquimbite, alunogen, paracoquimbite & \\
\hline \multirow[t]{2}{*}{ left side of the profile } & quartz, halotrichite g. & \\
\hline & gypsum, quartz & \\
\hline \multirow[t]{2}{*}{ spray-like aggregates } & szomolnokite, coquimbite, copiapite g. & alunogen, voltaite \\
\hline & tamarugite, quartz, coquimbite, gypsum, aubertite & \\
\hline \multicolumn{3}{|c|}{ PROFILE II } \\
\hline & alunogen, tamarugite, quartz & alum-(Na), tridymite, rostite \\
\hline \multicolumn{3}{|c|}{ PROFILE III } \\
\hline $\begin{array}{l}\text { lower part of the profile, } \\
\text { reddish aggregates }\end{array}$ & halotrichite g., copiapite g., gypsum, quartz & \\
\hline \multirow[t]{2}{*}{ upper part of the profile } & coquimbite, quartz, halotrichite g., alunogen, tamarugite & eugsterite \\
\hline & gypsum, quartz, sulphur (trace) & ardealite or sjögrenite \\
\hline \multicolumn{3}{|c|}{ PROFILE IV } \\
\hline \multirow[t]{2}{*}{ white aggregates } & alunogen, halotrichite g., meta-alunogen, quartz & \\
\hline & paracoquimbite, coquimbite, copiapite g. & \\
\hline \multicolumn{3}{|c|}{ PROFILE V } \\
\hline & alunogen, tamarugite, halotrichite g., quartz & metavoltine, nordstrandite \\
\hline \multicolumn{3}{|c|}{ Unknown source samples } \\
\hline \multirow[t]{15}{*}{ yellow masses } & copiapite g., quartz, alunogen & metavoltine \\
\hline & coquimbite, halotrichite $\mathrm{g}$. & tamarugite, ferrinatrite \\
\hline & copiapite g., quartz & aluminite, cristobalite \\
\hline & tamarugite, jarosite g. & alum-(Na) \\
\hline & tamarugite, halite, quartz, gypsum & \\
\hline & quartz, gypsum, jarosite g., metasideronatrite, illite & No. 30: boussingaultite \\
\hline & szomolnokite, coquimbite, halotrichite g. & rostite \\
\hline & tamarugite, gypsum & sassolite \\
\hline & halotrichite g., alunogen, coquimbite & \\
\hline & copiapite g., alunogen, metavoltine & zaherite, alum-(Na) \\
\hline & coquimbite, szomolnokite, quartz & \\
\hline & alunogen, copiapite g. & \\
\hline & coquimbite, quartz, halotrichite g., alunogen, tamarugite & eugsterite \\
\hline & szomolnokite, coquimbite, ferrinatrite & \\
\hline & coquimbite, alunogen, melanterite, szomolnokite, tamarugite & chalcoalumite \\
\hline "below the coal deposit" & quartz, paracoquimbite, coquimbite, alunogen, copiapite & mikasaite, vaterite \\
\hline \multirow[t]{3}{*}{ "directly on the coal" } & sideronatrite, metasideronatrite, copiapite g. & ferrinatrite \\
\hline & coquimbite, alunogen, szomolnokite & aubertite group \\
\hline & alunogen, coquimbite, szomolnokite ${ }^{2}$ & rostite \\
\hline
\end{tabular}

${ }^{1}$ - not observed in the re-analysed sample; ${ }^{2}-$ in subsample "b" 

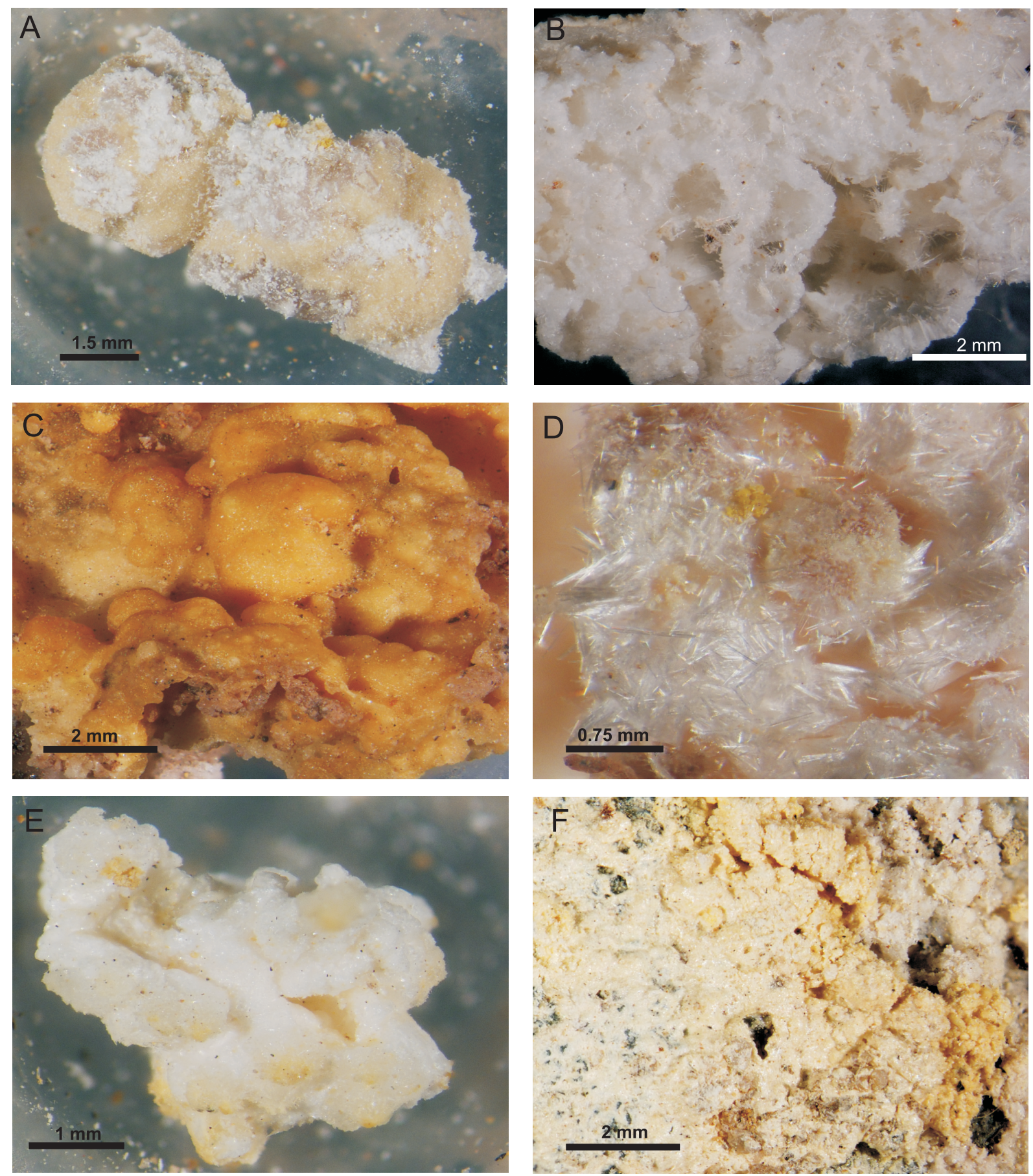

Fig. 2. General true-colour view of the example sulphate mixtures

A - white microbotryoidal rozenite with minor needles of gypsum on coquimbite (pale pinkish-violet) - copiapite group (yellow) matrix; B - compact mass of alunogen and meta-alunogen with needle-like halotrichite; $\mathbf{C}$ - botryoidal dark yellow copiapite group with minor compact alunogen; D - typical spray-like aggregates of halotrichite intergrown with minor colourless alunogen and pale pinkish coquimbite, the dark yellow mineral likely being metavoltine; $\mathbf{E}$ - tamarugite-dominant encrustation fragment with yellow jarosite; $\mathbf{F}$ - gypsum-jarosite-metasideronatrite encrustation on a sandstone chunk

gates (Fig. 3B, C, respectively). Tamarugite forms thin-tabular crystals reaching $8-12 \mu \mathrm{m}$, with pseudohexagonal and possibly octagonal outlines sometimes present. Typically lamellar crystals of alunogen can be $90 \mu \mathrm{m}$ long and always $<10 \mu \mathrm{m}$ thick. Metasideronatrite forms thick-tabular crystals, up to $15 \mu \mathrm{m}$ long and up to $\sim 3 \mu \mathrm{m}$ thick, that join into parallel or slightly bent aggregates usually comprising two individuals (Fig. 3E). The aggregates formed by copiapites, tamarugite and alunogen are thick, compact, socket-like and somewhat curved encrustations. 

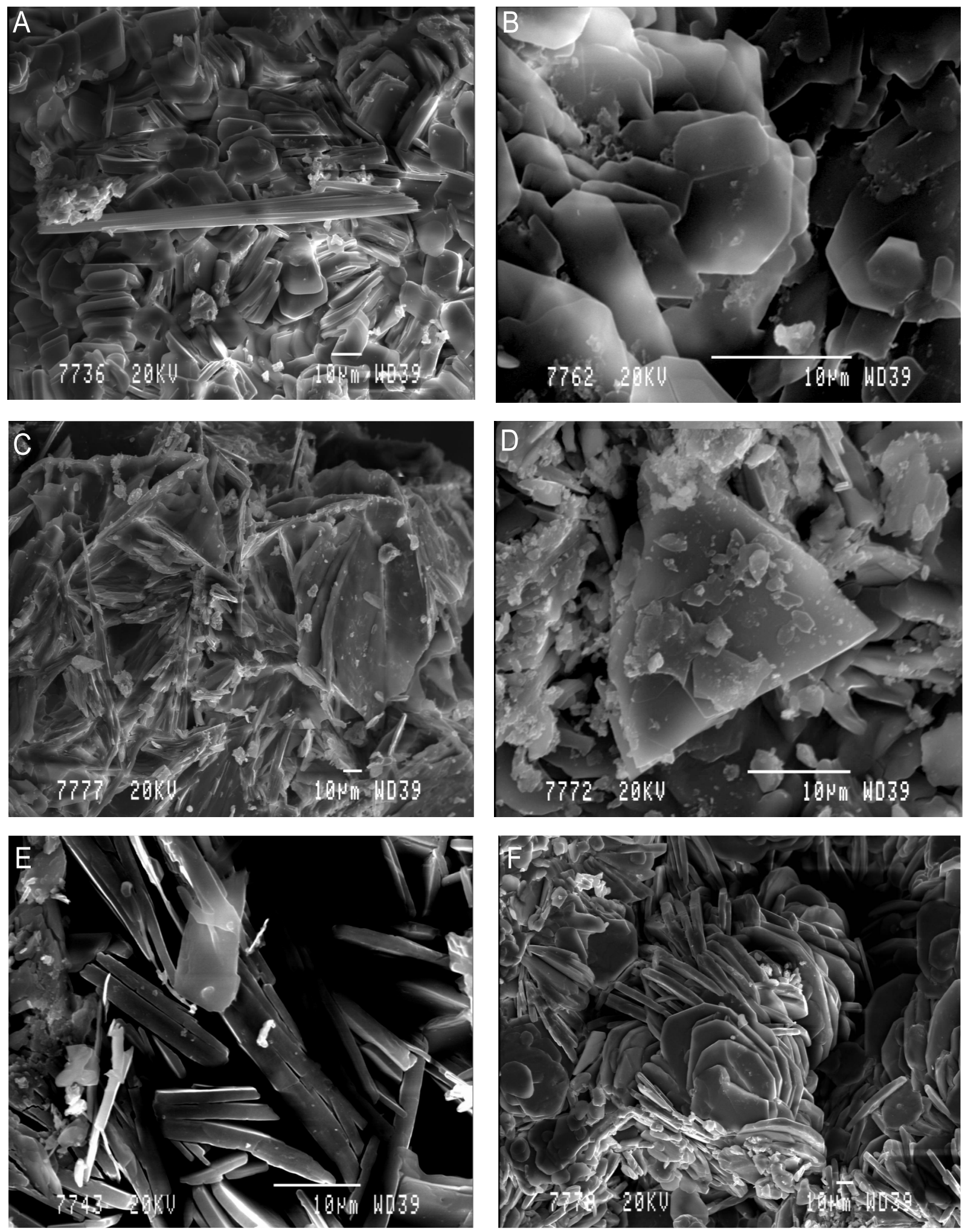

Fig. 3. Scanning Electron Microscopy secondary electron images of chosen sulphate minerals

A - fibrous halotrichite aggregates growing on rozenite crystals; B - Fe-bearing crystals of tamarugite; C - rosette-forming lamellar crystals of alunogen; D - triangle-shaped section of a paracoquimbite crystal; $\mathbf{E}$ - intergrown elongated crystals of metasideronatrite; F - rosette-like aggregates of bladed coquimbite crystals 


\section{CRYSTAL CHEMISTRY}

\section{SAMPLE Aou01}

The simple Fe sulphates in this sample proved to be impossible to be analysed quantitatively either due to their strong intergrowths with some Al-rich species or poor quality of the thin-section. However, an interesting additional phase was detected, with the empirical formula of $\left(\mathrm{Al}_{0.97} \mathrm{Fe}_{0.01} \mathrm{Na}_{0.01}\right)_{\Sigma 1.00}\left[\left(\mathrm{SO}_{4}\right)_{0.87}\left(\mathrm{SiO}_{4}\right)_{0.08}\left(\mathrm{AsO}_{4}\right)_{0.03}\right]_{\Sigma 0.98}\left[(\mathrm{OH})_{0.75}\right.$ $\left.\mathrm{Cl}_{0.07}\right]_{\Sigma_{0.82}} \cdot 7.94 \mathrm{H}_{2} \mathrm{O}(n=3)$. Its wt.\% contents are $0.00-2.15$ $\mathrm{As}_{2} \mathrm{O}_{5}, 0.25-3.92 \mathrm{SiO}_{2}, 14.98-19.73 \mathrm{Al}_{2} \mathrm{O}_{3}, 0.00-1.17 \mathrm{Fe}_{2} \mathrm{O}_{3}$, $0.00-1.22 \mathrm{Na}_{2} \mathrm{O}$, and $49.80-59.17 \mathrm{H}_{2} \mathrm{O}$. A single analysis also shows 0.79 wt. $\% \mathrm{SeO}_{3}$. Assuming the high water content being an error related to sample destruction under the electron beam, this phase is likely either rostite or jurbanite. The ideal composition of those polymorphic substances is $\mathrm{Al}\left(\mathrm{SO}_{4}\right)(\mathrm{OH}) \cdot 5 \mathrm{H}_{2} \mathrm{O}$.

\section{SAMPLE Aou02}

The copiapite group species is aluminocopiapite ( $n=12$; Table 2) in this case, as shown by the formula of $\left(\mathrm{Al}_{0.45} \mathrm{Ca}_{0.05} \mathrm{Sr}_{0.02} \mathrm{Na}_{0.01} \mathrm{~K}_{0.01}\right)_{\Sigma 0.54}\left(\mathrm{Fe}^{3+}{ }_{3.93} \mathrm{Ti}_{0.02}\right)_{\Sigma 3.95}\left(\mathrm{SO}_{4}\right)_{6.00}(\mathrm{OH})_{1.38}$ $23.80 \mathrm{H}_{2} \mathrm{O}$, normalized (by charge proportion) to $\left(\mathrm{Al}_{0.64} \mathrm{Ca}_{0.07} \mathrm{Sr}_{0.03} \mathrm{Na}_{0.01} \mathrm{~K}_{0.01}\right)_{\Sigma 0.76}\left(\mathrm{Fe}^{3+}{ }_{3.93} \mathrm{Ti}_{0.02}\right)_{\Sigma 3.95}\left(\mathrm{SO}_{4}\right)_{6.00}(\mathrm{OH})_{2.00}$ . $23.80 \mathrm{H}_{2} \mathrm{O}$. The latter formula may be expressed in terms of a mean end-members formula, as $\mathrm{Alc}_{84} \mathrm{Cac}_{9} \mathrm{Src}_{4} \mathrm{NcO}_{1} \mathrm{KcO}_{1} R_{1}$, where Alc = aluminocopiapite, $\mathrm{Cac}=$ calciocopiapite, and the remaining abbreviations being used for hypothetical Sr-, Na-, K-rich and remaining end-members. The latter ones are possibly represented mainly by a Ti-dominant end-member.

The associated coquimbite analyses $(n=11)$ were recalculated to $\left(\mathrm{Fe}_{1.72} \mathrm{Al}_{0.25} \mathrm{Mg}_{0.01} \mathrm{Sr}_{0.01}\right)_{\Sigma 2.00}\left(\mathrm{SO}_{4}\right)_{2.86} \cdot 10.74 \mathrm{H}_{2} \mathrm{O}$ or $\mathrm{Fe}_{1.00}\left(\mathrm{Fe}_{0.72} \mathrm{Al}_{0.25} \mathrm{Mg}_{0.01} \mathrm{Sr}_{0.01}\right)_{\Sigma 1.00}\left(\mathrm{SO}_{4}\right)_{2.86} \cdot 10.74 \mathrm{H}_{2} \mathrm{O}$. This expression corresponds to $\mathrm{Coq}_{74} \mathrm{Acq}_{26}$, where Acq stands for aluminocoquimbite, $\mathrm{FeAl}\left(\mathrm{SO}_{4}\right)_{3} \cdot 9 \mathrm{H}_{2} \mathrm{O}$.

Rozenite or a post-rozenite dehydrated counterpart was also found associated. Its empirical formula $(n=9)$ is: $\left(\mathrm{Fe}_{0.93} \mathrm{Mg}_{0.03} \mathrm{~K}_{0.02} \mathrm{Ca}_{0.01} \mathrm{Mn}_{0.01} \mathrm{Al}_{0.01}\right)_{\Sigma 1.01}\left[\left(\mathrm{SO}_{4}\right)_{1.11}\left(\mathrm{AsO}_{4}\right)_{0.01}\right]_{\Sigma 1.12}$. . $4.28 \mathrm{H}_{2} \mathrm{O}$. Assuming $\mathrm{K}$ and $\mathrm{Al}$ as impurities, this formula may be transformed to a proposal of an end-members formula, e.g. $\mathrm{RoZ}_{95} \mathrm{Sta}_{3} \mathrm{lle}_{1} \mathrm{CrO}_{1}$, where "Sta" is for starkeyite $\left(\mathrm{MgSO}_{4} \cdot 4 \mathrm{H}_{2} \mathrm{O}\right)$, "lle" is for ilesite $\left(\mathrm{MnSO}_{4} \cdot 4 \mathrm{H}_{2} \mathrm{O}\right)$ and "Cro" is for a hypothetical Ca-dominant member. The lowered sulphur content and surplus water content are related to the occurrence of part of $\mathrm{Fe}$ as $\mathrm{Fe}^{3+}$ and possibly also to the sample destruction under the electron beam and intimate intergrowths of the minerals.

\section{SAMPLE Aou10}

Only a single analysis could be attributed to aluminocopiapite-magnesiocopiapite, although still intimately intergrown with gypsum. After deducting gypsum admixture, the first-step empirical formula may be approximated as $\left(\mathrm{Al}_{0.22} \mathrm{Mg}_{0.17} \mathrm{~K}_{0.10}\right)_{\Sigma 0.49} \mathrm{Fe}_{4.00}\left[\left(\mathrm{SO}_{4}\right)_{5.32}\left(\mathrm{SiO}_{4}\right)_{0.22}\right]_{\Sigma 5.54}(\mathrm{OH})_{1.58} \cdot x \mathrm{H} 2 \mathrm{O}$. Assumption of the $\mathrm{Al}: \mathrm{Mg}$ ratio being correct and equal to 1.29 $(0.22 / 0.17)$ suggests that $\mathrm{Al}^{3+}=1.29 \cdot \mathrm{Mg}^{2+}$. The lacking positive charge being 2 (positive charge from $\mathrm{Fe}^{3+}$ is 12 , negative charge from the anions is 14) allows writing the formula: $3 \mathrm{Al}+2 \mathrm{Mg}=2$. The above considerations and removal of the likely $\mathrm{K}$ and $\mathrm{Si}$ impurity lead to $\left(\mathrm{Al}_{0.44} \mathrm{Mg}_{0.34}\right)_{\Sigma 0.78} \mathrm{Fe}_{4.00}\left(\mathrm{SO}_{4}\right)_{6}(\mathrm{OH})_{2} \cdot x \mathrm{H} 2 \mathrm{O}$.
SAMPLE Aou14

Application of the less destructive EDS beam to analyse the Na-rich sulphates, as opposed to the results obtained by Kruszewski (2013), proved not to give the correct $\mathrm{Na}$ apfu values. In such case the true Na content may be calculated by difference. However, no $\mathrm{K}$ and $\mathrm{Ca}$ admixture was found (Table 3 ). Tamarugite from sample Aou14 may thus be characterized in terms of its crystal chemistry by the following formula $(n=22$, factor based on $\mathrm{Al}+\mathrm{Fe}=2)$ :

$\mathrm{Na}\left(\mathrm{Al}_{0.98} \mathrm{Fe}^{3+}{ }_{0.02}\right)_{\Sigma 1.00}\left[\left(\mathrm{SO}_{4}\right)_{1.89}\left(\mathrm{AsO}_{4}\right)_{0.05}\left(\mathrm{SiO}_{4}\right)_{0.01}\right]_{\Sigma 1.95} \mathrm{Cl}_{0.01}$. $7.53 \mathrm{H}_{2} \mathrm{O}$. Such formula leads to the mean end-member representation of $\mathrm{Tmr}_{98} \mathrm{Ama}_{2}$ (the minor and hypothetic As- and Si-bearing end-members omitted), where "Ama" corresponds to amarillite, $\mathrm{NaFe}\left(\mathrm{SO}_{4}\right)_{2} \cdot 6 \mathrm{H}_{2} \mathrm{O}$. A single analysis corresponds to a similar Fe-rich species which can possibly be expressed as $\mathrm{Na}_{0.33}(\mathrm{Fe}, \mathrm{Al})\left(\mathrm{SO}_{4}\right)(\mathrm{OH}) \cdot x \mathrm{H} 2 \mathrm{O}$ or $\mathrm{Na}(\mathrm{Fe}, \mathrm{Al})\left(\mathrm{SO}_{4}\right)(\mathrm{OH})_{2} \cdot x \mathrm{H} 2 \mathrm{O}$.

\section{SAMPLE Aou20}

Only two of six analytical points corresponding to the copiapite group could be chosen for description of the mean formula. The latter, fitting to copiapite, is

$\left(\mathrm{Fe}^{2+}{ }_{0.64} \mathrm{Al}_{0.18} \mathrm{~K}_{0.08} \mathrm{Ca}_{0.05} \mathrm{Co}_{0.05}\right)_{\Sigma 1.00} \mathrm{Fe}_{4.00}\left[\left(\mathrm{SO}_{4}\right)_{5.97}\left(\mathrm{SiO}_{4}\right)_{0.03}\right]_{\Sigma 6.00}(\mathrm{OH})_{2}$ . $x \mathrm{H} 2 \mathrm{O} x \mathrm{H}_{2} \mathrm{O}$ (the $x$ value taken directly from the analysis recasting is 31.37). This formula corresponds to the following mean wt.\% concentrations: $32.71 \mathrm{SO}_{3}, 25.93 \mathrm{Fe}_{2} \mathrm{O}_{3}, 0.61 \mathrm{Al}_{2} \mathrm{O}_{3}, 0.24$ $\mathrm{CoO}, 0.21 \mathrm{CaO}, 0.27 \mathrm{~K}_{2} \mathrm{O}$, and $39.91 \mathrm{H}_{2} \mathrm{O}$. The reported formula is related to the mean end-member representation of

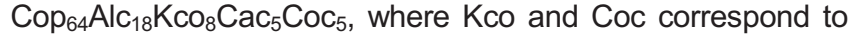
hypothetical K- and Co-dominant end-members. The formula, in its normalized (the $A$ site total is originally 1.62) version, is $\left(\mathrm{Fe}^{2+}{ }_{0.70} \mathrm{Al}_{0.25} \mathrm{Ca}_{0.01} \mathrm{~K}_{0.01}\right)_{\Sigma 1.00} \mathrm{Fe}_{4.00}\left[\left(\mathrm{SO}_{4}\right)_{5.99}\left(\mathrm{SiO}_{4}\right)_{0.01}\right]_{\Sigma 6.00}(\mathrm{OH})_{2}$. - $28.77 \mathrm{H}_{2} \mathrm{O}$ and is based on 12 analyses. The normalization basis is the $A$ site occupancy of 1 . It corresponds to the end-member formula of $\mathrm{Cop}_{70} \mathrm{Alc}_{18} \mathrm{Cac}_{1} \mathrm{KcO}_{1}$.

\section{SAMPLE Aou22}

The example empirical formula for aluminocoquimbite $(n=$ 1) may be approximated as $\mathrm{Fe}_{0.99}\left(\mathrm{Al}_{0.73} \mathrm{Sr}_{0.09} \mathrm{Na}_{0.08} \mathrm{Ga}_{0.06} \mathrm{~K}_{0.02}\right.$ $\left.\mathrm{Ti}_{0.01}\right)_{\Sigma 0.99}\left(\mathrm{SO}_{4}\right)_{2.83} \cdot x \mathrm{H} 2 \mathrm{O}$, corresponding to the following $\mathrm{wt} \%$ proportions: $14.40 \mathrm{SO}_{3}, 0.15 \mathrm{TiO}_{2}, 10.13 \mathrm{Fe}_{2} \mathrm{O}_{3}, 4.10 \mathrm{Al}_{2} \mathrm{O}_{3}$, $0.59 \mathrm{Ga}_{2} \mathrm{O}_{3}, 1.03 \mathrm{SrO}, 0.14 \mathrm{~K}_{2} \mathrm{O}$, and $0.84 \mathrm{Na}_{2} \mathrm{O}$. Neglecting the supposed $\mathrm{Sr}$-, $\mathrm{K}$-, $\mathrm{Na}$ - and Ti-bearing members, the latter expression corresponds to $\mathrm{Acq}_{93} \mathrm{Gcq}_{7}$, where "Gcq" stands for a hypothetical Ga-rich end-member. The normalized empirical formula of the coexisting tamarugite $(n=2)$ is $\left(\mathrm{Na}_{0.86} \mathrm{Sr}_{0.10} \mathrm{Ca}_{0.05}\right)_{\Sigma 0.99}\left(\mathrm{Al}_{0.87} \mathrm{Fe}_{0.13}\right)_{\Sigma 1.00}\left[\left(\mathrm{SO}_{4}\right)_{1.91}\left(\mathrm{SeO}_{3}\right)_{0.05}\left(\mathrm{SiO}_{4}\right)\right.$ $0.03]_{\Sigma 1.99} \mathrm{Cl}_{0.12} \cdot 5.16 \mathrm{H}_{2} \mathrm{O}$ (originally 35.56 molecules $\mathrm{H}_{2} \mathrm{O}$ and $0.18 \mathrm{apfu} \mathrm{Na}$ ). It corresponds to mean (wt.\%) $27.24 \mathrm{SO}_{3}$, $1.19 \mathrm{SeO}_{3}, 0.47 \mathrm{SiO}_{2}, 3.15 \mathrm{Fe}_{2} \mathrm{O}_{3}, 13.27 \mathrm{Al}_{2} \mathrm{O}_{3}, 0.63 \mathrm{SrO}$, $0.12 \mathrm{CaO}, 1.69 \mathrm{Na}_{2} \mathrm{O}$, and $50.52 \mathrm{H}_{2} \mathrm{O}$. Assuming $\mathrm{Cl}$ not entering the structure, the above formula may be redrawn as $\mathrm{Tmr}_{76} \mathrm{Ama}_{11} \mathrm{Srt}_{9} \mathrm{Cat}_{4} \mathrm{Sra}_{1} \mathrm{Caa}_{1}$, where "Srt", "Cat", "Sra" and "Caa" correspond to some Sr-, Ca- HEMs. A single analysis of alunogen (9.87 wt. \% SO $3,6.73$ wt. $\% \mathrm{SeO}_{3}, 14.98$ wt. \% $\mathrm{Al}_{2} \mathrm{O}_{3}, 0.28$ wt. \% SrO, 0.15 wt. \% $\mathrm{CaO}, 0.17$ wt. $\% \mathrm{Na}_{2} \mathrm{O}$, and 66.69 wt.\% $\left.\mathrm{H}_{2} \mathrm{O}\right)$ recasts to $\left(\mathrm{Al}_{2.00} \mathrm{Na}_{0.04} \mathrm{Ca}_{0.02}\right.$ $\left.\mathrm{Sr}_{0.02}\right)_{\Sigma 1.07}\left[\left(\mathrm{SO}_{4}\right)_{2.14}\left(\mathrm{SeO}_{3}\right)_{0.92}\right]_{\Sigma 3.06} \cdot 17 \mathrm{H}_{2} \mathrm{O}$ (ideal water content assumed). The derived suggested end-member representation is $\mathrm{Aln}_{70} \mathrm{Sea}_{30}$, where "Sea" corresponds to a Se-rich HEM. 
Results of chemical analyses (in wt.\%) and crystallographic data for sulphate minerals of sample Aou02

\begin{tabular}{|c|c|c|c|c|c|c|c|c|c|c|c|c|c|c|c|c|c|c|}
\hline & 1 & 2 & 3 & 4 & 5 & 6 & 7 & 8 & 9 & 10 & 11 & 12 & 13 & 14 & 15 & 16 & 17 & 18 \\
\hline $\mathrm{As}_{2} \mathrm{O}_{5}{ }^{1)}$ & & & & & & & & & & & & & & & & & & \begin{tabular}{|l|}
0.64 \\
\end{tabular} \\
\hline $\mathrm{SO}_{3}$ & 36.12 & 39.07 & 36.92 & 35.87 & 33.70 & 40.55 & 42.45 & 39.73 & 39.31 & 40.24 & 41.09 & 39.38 & 32.00 & 35.60 & 33.83 & 31.55 & 36.63 & 37.73 \\
\hline $\mathrm{SiO}_{2}$ & & & & & & & & & & & & & & & 0.30 & \begin{tabular}{|l|}
0.57 \\
\end{tabular} & & \\
\hline $\mathrm{Ga}_{2} \mathrm{O}_{3}$ & & & & & & & & & & & & & & & 0.62 & & & \\
\hline $\mathrm{Fe}_{2} \mathrm{O}_{3}$ & 23.69 & 24.57 & 24.99 & 24.67 & 23.13 & 27.56 & 26.01 & 23.70 & 22.54 & 24.70 & 25.66 & 24.73 & 23.69 & 26.88 & 26.78 & 24.30 & 31.42 & 31.52 \\
\hline $\mathrm{Al}_{2} \mathrm{O}_{3}$ & 2.51 & 2.02 & 1.57 & 1.56 & 1.62 & & 2.37 & 2.43 & 2.36 & 1.90 & 2.25 & 1.82 & 1.95 & 1.32 & & & 0.83 & 0.58 \\
\hline $\mathrm{MnO}$ & & & & & & & & & & & & & & 0.26 & 0.44 & & 0.31 & 0.23 \\
\hline $\mathrm{MgO}$ & & & & & & & & & & & & & & 0.25 & & & & \\
\hline SrO & & 0.19 & 0.23 & 0.20 & 0.18 & & & 0.33 & 0.30 & 0.24 & 0.25 & 0.34 & & 0.16 & & \begin{tabular}{|l|}
0.27 \\
\end{tabular} & & 0.20 \\
\hline $\mathrm{CaO}$ & \begin{tabular}{|l|l|}
0.14 \\
\end{tabular} & 1.51 & 0.25 & & 0.13 & 0.33 & & & 0.13 & & & 0.14 & & 0.54 & 0.25 & \begin{tabular}{|l|}
0.38 \\
\end{tabular} & & \\
\hline $\mathrm{K}_{2} \mathrm{O}$ & & & & & & 0.48 & & 0.08 & 0.07 & 0.10 & & & & & 3.21 & 2.34 & & \\
\hline $\mathrm{Na}_{2} \mathrm{O}$ & \begin{tabular}{|l|}
0.27 \\
\end{tabular} & & & & & & & & & & & & & & & & & \\
\hline$\Sigma$ & 62.73 & 67.36 & 63.96 & 62.30 & 58.67 & 69.18 & 70.83 & \begin{tabular}{|l|}
66.27 \\
\end{tabular} & 64.71 & 67.18 & 69.25 & 66.41 & 57.64 & 65.31 & 65.43 & 59.41 & 69.19 & 70.90 \\
\hline $\mathrm{H}_{2} \mathrm{O}^{2)}$ & 37.27 & 32.64 & 36.04 & 37.70 & 41.24 & 30.82 & 29.17 & 33.73 & 35.29 & 32.82 & 30.75 & 33.59 & 42.36 & 34.69 & 34.57 & 40.59 & 30.81 & 29.10 \\
\hline \multicolumn{19}{|c|}{ apfu / molecular pfu } \\
\hline As & & & & & & & & & & & & & & & & & & 0.01 \\
\hline$S$ & 6.00 & 6.00 & 6.00 & 6.00 & 6.00 & 6.00 & 2.85 & 2.84 & 2.93 & 2.86 & 2.79 & 2.80 & 1.19 & 1.16 & 1.00 & 1.08 & 1.09 & 1.14 \\
\hline $\mathrm{Si}$ & & & & & & & & & & & & & & & 0.01 & 0.03 & & \\
\hline $\mathrm{Fe}$ & 3.95 & 3.78 & 4.07 & 4.14 & 4.13 & 4.09 & 1.75 & 1.70 & 1.68 & 1.76 & 1.75 & 1.76 & 0.89 & 0.88 & 0.80 & 0.84 & 0.94 & 0.95 \\
\hline $\mathrm{Al}$ & 0.65 & 0.49 & 0.40 & 0.41 & 0.45 & & 0.25 & 0.27 & 0.28 & 0.21 & 0.24 & 0.20 & 0.11 & 0.07 & & & & \\
\hline $\mathrm{Mn}$ & & & & & & & & & & & & & & 0.01 & 0.01 & & 0.01 & 0.01 \\
\hline $\mathrm{Mg}$ & & & & & & & & & & & & & & 0.02 & & & 0.05 & 0.03 \\
\hline $\mathrm{Sr}$ & & 0.02 & 0.03 & 0.03 & 0.02 & & & 0.02 & 0.02 & 0.01 & 0.01 & 0.02 & & & & 0.01 & & \\
\hline $\mathrm{Ca}$ & 0.03 & 0.33 & 0.06 & & 0.03 & 0.07 & & & 0.01 & & & 0.01 & & 0.03 & 0.01 & 0.02 & & \\
\hline $\mathrm{K}$ & & & & & & 0.12 & & 0.01 & 0.01 & 0.01 & & & & & 0.16 & 0.14 & & \\
\hline $\mathrm{Na}$ & 0.12 & & & & & & & & & & & & & & & & & \\
\hline $\mathrm{H}_{2} \mathrm{O}$ & 27.51 & 22.78 & 26.03 & 28.03 & 32.63 & 20.27 & 8.70 & 10.72 & 11.68 & 10.38 & 9.28 & 10.62 & 7.02 & 5.02 & 4.56 & 6.20 & 4.09 & 3.90 \\
\hline \multicolumn{19}{|c|}{ unit cell parameters } \\
\hline$a[\AA]$ & \multicolumn{7}{|c|}{$7.42(2)$} & \multicolumn{6}{|c|}{$10.970(14)$} & \multicolumn{5}{|c|}{ 3) } \\
\hline$b[\AA]$ & \multicolumn{7}{|c|}{$18.89(4)$} & & & & & & \\
\hline$c[\AA \AA]$ & \multicolumn{7}{|c|}{$7.46(2)$} & & & & & & & & & & & \\
\hline$\alpha\left[\left[^{0}\right]\right.$ & \multicolumn{7}{|c|}{$90.60(12)$} & \multicolumn{6}{|c|}{$17.00(33)$} & & & & & \\
\hline$\beta\left[^{0}\right]$ & \multicolumn{7}{|c|}{$102.96(14)$} & & & & & & & & & & & \\
\hline$\gamma\left[{ }^{0}\right]$ & \multicolumn{7}{|c|}{$98.00(11)$} & & & & & & & & & & & \\
\hline $\begin{array}{l}\mathrm{R}_{\mathrm{wp}_{4}} \\
\left.[\%]^{4}\right)\end{array}$ & \multicolumn{7}{|c|}{31.55} & \multicolumn{6}{|c|}{31.55} & & & & & \\
\hline $\begin{array}{l}\text { GOF } \\
{[\%]}\end{array}$ & \multicolumn{7}{|c|}{1.97} & \multicolumn{6}{|c|}{1.97} & & & & & \\
\hline
\end{tabular}

Analyses 1-6 - copiapite group; analyses 7-12- coquimbite group; analyses $13-18$ - rozenite; ${ }^{1)}-\mathrm{P}, \mathrm{As}, \mathrm{Se}, \mathrm{Ti}, \mathrm{Cr}, \mathrm{Zn}, \mathrm{Cu}, \mathrm{Ni}$ and $\mathrm{Co}$ were analysed but not observed; zero values are not shown; ${ }^{2)}$ - calculated as $100-\Sigma ;{ }^{3)}$ - unit cell parameters could not be calculated due to low content of rozenite; ${ }^{4)}$ - refinement statistics: $R_{\mathrm{wp}}$ - residual weighted-pattern, GOF - goodness of fit $\left(\chi^{2}\right)$

\section{SAMPLE Aou29}

The Aou29 coquimbite composition (Table 4) may be presented by two empirical formulas, one based on 5 analytical points and the other corresponding to $n=19$. The formulas are, respectively,

$\mathrm{Fe}_{1.00}\left(\mathrm{Fe}_{0.50} \mathrm{Al}_{0.43} \mathrm{Na}_{0.02} \mathrm{Ti}_{0.02} \mathrm{Ga}_{0.01} \mathrm{Cu}_{0.01}\right)_{\Sigma 0.99}\left[\left(\mathrm{SO}_{4}\right)_{2.82}\left(\mathrm{SiO}_{4}\right)_{0.06}\right.$ $\left.\left(\mathrm{AsO}_{4}\right)_{0.02}\right]_{22.90} \cdot 9 \mathrm{H}_{2} \mathrm{O}$ (ideal water content assumed), and $\mathrm{Fe}_{1.00}\left(\mathrm{Fe}_{0.50} \mathrm{Al}_{0.43} \mathrm{Ti}_{0.03} \mathrm{Na}_{0.01} \mathrm{Ca}_{0.01} \mathrm{Mg}_{0.01} \mathrm{Ga}_{0.01}\right)_{\Sigma 1.00}\left[\left(\mathrm{SO}_{4}\right)_{2.84}\right.$ $\left.\left(\mathrm{SiO}_{4}\right)_{0.06}\left(\mathrm{AsO}_{4}\right)_{0.02}\right]_{\Sigma 2.92} \cdot 5.43 \mathrm{H}_{2} \mathrm{O}$ (original calculated water content).
Szomolnokite analyses (Table 4) recast to the following empirical formula $(n=7)$ :

$\left(\mathrm{Fe}_{0.85} \mathrm{Al}_{0.06} \mathrm{Na}_{0.05} \mathrm{Mg}_{0.02} \mathrm{Ti}_{0.01} \mathrm{Ca}_{0.01} \mathrm{Ga}_{0.01}\right)_{\Sigma 1.01}\left[\left(\mathrm{SO}_{4}\right)_{0.93}\left(\mathrm{SiO}_{4}\right)_{0.03}\right]_{\Sigma 0.96}$ . $1.06 \mathrm{H}_{2} \mathrm{O}$. The identity of the phase with szomolnokite may be confirmed by relatively enriched magnesium. The presence of silicon in the mineral structure is unsure due to rather strong variation of its content. A single analysis (no. 15 in Table 4) corresponds to a likely melanterite-derived mineral, now closing to ferrohexahydrite:

$\left(\mathrm{Fe}_{0.74} \mathrm{Al}_{0.11} \mathrm{Ti}_{0.11} \mathrm{Zn}_{0.02} \mathrm{Mg}_{0.01} \mathrm{Ca}_{0.01}\right)_{\Sigma 1.01}\left[\left(\mathrm{SO}_{4}\right)_{1.14}\left(\mathrm{SiO}_{4}\right)_{0.02}\right]_{\Sigma 1.16}$ $\mathrm{Cl}_{0.01} \mathrm{C} 6.08 \mathrm{H}_{2} \mathrm{O}$. In this case the $\mathrm{Zn}$ enrichment seem to confirm the identification: bianchite, $\mathrm{ZnSO}_{4} \cdot 6 \mathrm{H}_{2} \mathrm{O}$ is a member of the 
Results of chemical analyses (in wt.\%) of tamarugite (analyses 1-10) and a related phase (analysis 11) of sample Aou14

\begin{tabular}{|c|c|c|c|c|c|c|c|c|c|c|c|}
\hline & 1 & 2 & 3 & 4 & 5 & 6 & 7 & 8 & 9 & 10 & 11 \\
\hline $\mathrm{As}_{2} \mathrm{O}_{5}{ }^{1)}$ & 1.57 & 1.51 & 1.33 & 1.45 & 1.32 & 1.44 & 1.28 & 1.57 & 1.49 & 1.36 & \\
\hline $\mathrm{SO}_{3}$ & 44.19 & 43.75 & 42.00 & 43.09 & 42.74 & 42.60 & 39.47 & 43.90 & 42.99 & 42.74 & 19.07 \\
\hline $\mathrm{SiO}_{2}$ & 0.27 & 0.15 & & 0.39 & 0.19 & & 0.12 & 0.18 & 0.15 & 0.14 & 1.32 \\
\hline $\mathrm{Ga}_{2} \mathrm{O}_{3}$ & & & & & & & & & & & 1.05 \\
\hline $\mathrm{Fe}_{2} \mathrm{O}_{3}$ & 0.27 & 0.54 & & 0.26 & 0.24 & 0.58 & 0.50 & 0.34 & 0.43 & 0.32 & 10.40 \\
\hline $\mathrm{Al}_{2} \mathrm{O}_{3}$ & 14.00 & 13.26 & 12.81 & 13.51 & 12.82 & 13.08 & 12.61 & 14.01 & $\begin{array}{c}13.451 \\
4.60 \\
\end{array}$ & 14.60 & 4.57 \\
\hline $\mathrm{CoO}$ & & & & & & & & 0.21 & & & \\
\hline $\mathrm{MgO}$ & & & & & & & & & & & 0.30 \\
\hline $\mathrm{CaO}$ & & & 0.08 & & 0.14 & & & 0.08 & & & 0.13 \\
\hline $\mathrm{Na}_{2} \mathrm{O}$ & 4.22 & 4.07 & 3.77 & 4.15 & 4.24 & 3.20 & 2.94 & 3.44 & 3.00 & 2.77 & 1.62 \\
\hline$\Sigma$ & 64.68 & 63.41 & 60.21 & 63.01 & 61.80 & 61.06 & 57.31 & 63.94 & 61.65 & 62.13 & 37.81 \\
\hline $\mathrm{H}_{2} \mathrm{O}^{2)}$ & 35.32 & 36.59 & 39.79 & $\begin{array}{c}36.993 \\
8.20 \\
\end{array}$ & 38.20 & 38.94 & 42.69 & 36.06 & 38.35 & 37.87 & 62.19 \\
\hline \multicolumn{12}{|c|}{ apfu / molecules pfu } \\
\hline As & 0.05 & 0.05 & 0.05 & 0.05 & 0.05 & 0.05 & 0.04 & 0.05 & 0.05 & 0.04 & \\
\hline$S$ & 1.99 & 2.05 & 2.09 & 2.01 & 2.10 & 2.02 & 1.93 & 1.95 & 1.99 & 1.84 & 1.00 \\
\hline Si & 0.02 & 0.01 & & 0.02 & 0.01 & & 0.01 & 0.01 & 0.01 & 0.01 & 0.02 \\
\hline $\mathrm{Ga}$ & & & & & & & & & & & 0.05 \\
\hline $\mathrm{Fe}$ & 0.01 & 0.03 & & 0.01 & 0.01 & 0.03 & 0.02 & 0.02 & 0.02 & 0.01 & 0.55 \\
\hline $\mathrm{Al}$ & 0.99 & 0.97 & 1.00 & 0.99 & 0.99 & 0.97 & 0.97 & 0.97 & 0.98 & 0.99 & 0.38 \\
\hline Co & & & & & & & & 0.01 & & & \\
\hline $\mathrm{Mg}$ & & & & & & & & & & & 0.03 \\
\hline $\mathrm{Ca}$ & & & 0.01 & & 0.01 & & & 0.01 & & & 0.01 \\
\hline $\mathrm{Na}$ & 0.49 & 0.49 & 0.48 & 0.50 & 0.54 & 0.39 & 0.37 & 0.39 & 0.36 & 0.31 & 0.22 \\
\hline $\mathrm{H}_{2} \mathrm{O}$ & 7.05 & 7.61 & 8.79 & 7.65 & 8.33 & 8.19 & 9.27 & 7.10 & 7.91 & $7.24^{3)}$ & 14.47 \\
\hline
\end{tabular}

1) - P, Se, Ti, Cr, Mn, Zn, Cu, Ni, Sr, Ba and K were analysed but not observed; zero values are not shown; ${ }^{2)}-\mathrm{calculated}^{2}$ as $100-\Sigma ;^{3)}$ - unit cell parameters could not be calculated due to low content of the mineral in the sample

hexahydrite group and may contain elevated Fe amounts (e.g., Palache et al., 1951). There are also two additional analyses (nos. 16 and 17; Table 4), one corresponding to either slightly aluminous parabutlerite or possibly a $\mathrm{FeSO}_{4} \cdot 3 \mathrm{H}_{2} \mathrm{O}$ phase, and the other to a $\mathrm{Zn}$-dominant phase close to $(\mathrm{Zn}, \mathrm{Fe})_{4}\left(\mathrm{SO}_{4}\right)_{2}(\mathrm{OH})_{3}$ . $7.5 \mathrm{H}_{2} \mathrm{O}$. The identity of a Ti-rich phase (with $7.63 \mathrm{wt} \% \% \mathrm{TiO}_{2}$; last analysis in Table 4) is unclear, as it may be related to an intergrowth.

\section{SAMPLE AOU33}

Analysing sideronatrite and metasideronatrite is difficult due to their $\mathrm{Na}$ content and delicate nature of these species. The minerals proved to be even more difficult to analyse than tamarugite. For most of the analyses the $\mathrm{Na}_{2} \mathrm{O}$ wt.\% content was measured at the beginning, in the middle and just before the end of the 60 -second-long analysis counts. A diagram juxtaposing analysis times and wt.\% $\mathrm{Na}_{2} \mathrm{O}$ usually shows non-linear, polynomial ("half-parabola") trends with attributed linear $r^{2}$ in the 0.61-0.87 range. However, two analyses show linear trends with $r^{2}$ values being 0.97 and 0.98 .

Results of analyses of sulphates from sample Aou33 are juxtaposed in Table 5. An empirical formula after normalizing to apfu $\mathrm{Na}$ being 2 and back-calculation of wt. $\% \mathrm{Na}_{2} \mathrm{O}$ and wt. $\%$ $\mathrm{H}_{2} \mathrm{O}$ is $\mathrm{Na}_{2.00}\left(\mathrm{Fe}_{0.88} \mathrm{Al}_{0.08} \mathrm{Ga}_{0.04}\right)_{\Sigma 1.00}\left(\mathrm{SO}_{4}\right)_{2.00}(\mathrm{OH})_{1.00} \cdot 2.18 \mathrm{H}_{2} \mathrm{O}$
( $n=25)$. This formula may, however, be deconvoluted, based on the newly calculated (by charge balance) apfu $\mathrm{H}$ to the composition of:

1. $\left(\mathrm{Na}_{1.99} \mathrm{Sr}_{0.01}\right)_{\Sigma 2.00}\left(\mathrm{Fe}_{0.79} \mathrm{Al}_{0.17} \mathrm{Ga}_{0.05}\right)_{\Sigma 1.01}\left(\mathrm{SO}_{4}\right)_{2.00}(\mathrm{OH})_{1.04}$. . 8. $14 \mathrm{H}_{2} \mathrm{O}(n=3$, apfu $\mathrm{H}>10$, analyses $1-3$ in Table 5), possibly corresponding to sideronatrite $\left(\mathrm{Sdn}_{78} \mathrm{Asd}_{17} \mathrm{Gsd}_{5}\right)$ with analysis-driven enlarged water content, and

2. $\mathrm{Na}_{2.00}\left(\mathrm{Fe}_{0.89} \mathrm{Al}_{0.07} \mathrm{Ga}_{0.04}\right)_{\Sigma 1.00}\left(\mathrm{SO}_{4}\right)_{2.00}(\mathrm{OH})_{1.00} \cdot 1.65 \mathrm{H}_{2} \mathrm{O}$ corresponding to metasideronatrite, $\mathrm{Msd}_{89} \mathrm{Ams}_{7} \mathrm{Gms}_{4}(n=21$, example analyses Nos. 4-9 in Table 5; Ams and Gms indicative of Al- and Ga-dominant HEMs). Three additional analyses (Nos. 10-12 in Table 5) may correspond to ferrinatrite, $\mathrm{Na}_{3.00}\left(\mathrm{Fe}_{0.75} \mathrm{Al}_{0.20} \mathrm{Ga}_{0.04}\right)_{20.99}\left(\mathrm{SO}_{4}\right)_{2.99} \cdot x \mathrm{H} 2 \mathrm{O}$ (the ideal $n=3$ ), mean end-member composition being $\mathrm{Frn}_{76} \mathrm{Amn}_{20} \mathrm{Gan}_{4}$ (HEMs: "Amn" - "aluminonatrite", "Gan" - "gallionatrite"). Associated with the (meta)sideronatrite is minor copiapite, the corresponding empirical formula $(n=6$; example analyses in Table 5 have Nos. 13-15) being $\left(\mathrm{Fe}^{2+}{ }_{0.56} \mathrm{Mg}_{0.45}\right)_{\Sigma 1.01}\left(\mathrm{Fe}_{3.99} \mathrm{Ti}_{0.01}\right)_{\Sigma 4.00}\left[\left(\mathrm{SO}_{4}\right)_{5.96}\left(\mathrm{AsO}_{4}\right)_{0.02}\right.$ $\left.\left(\mathrm{SiO}_{4}\right)_{0.01}\right]_{55.99}(\mathrm{OH})_{2.02} \cdot 27.58 \mathrm{H}_{2} \mathrm{O}$, related to $\mathrm{Cop}_{54} \mathrm{MgC}_{45} \mathrm{Tic}_{1}$ ("Tic" stands for a Ti-dominant HEM). This copiapite group representative is thus interesting among the previous ones, as it is very rich in the magnesiocopiapite molecule. The above species may also be associated with a phase which, as in the previous sample, may possibly be derived from melanterite via its dehydration and partial oxidation. Its empirical formula $(n=7$; 
Results of chemical analyses (in wt.\%) of sulphate minerals of sample Aou29

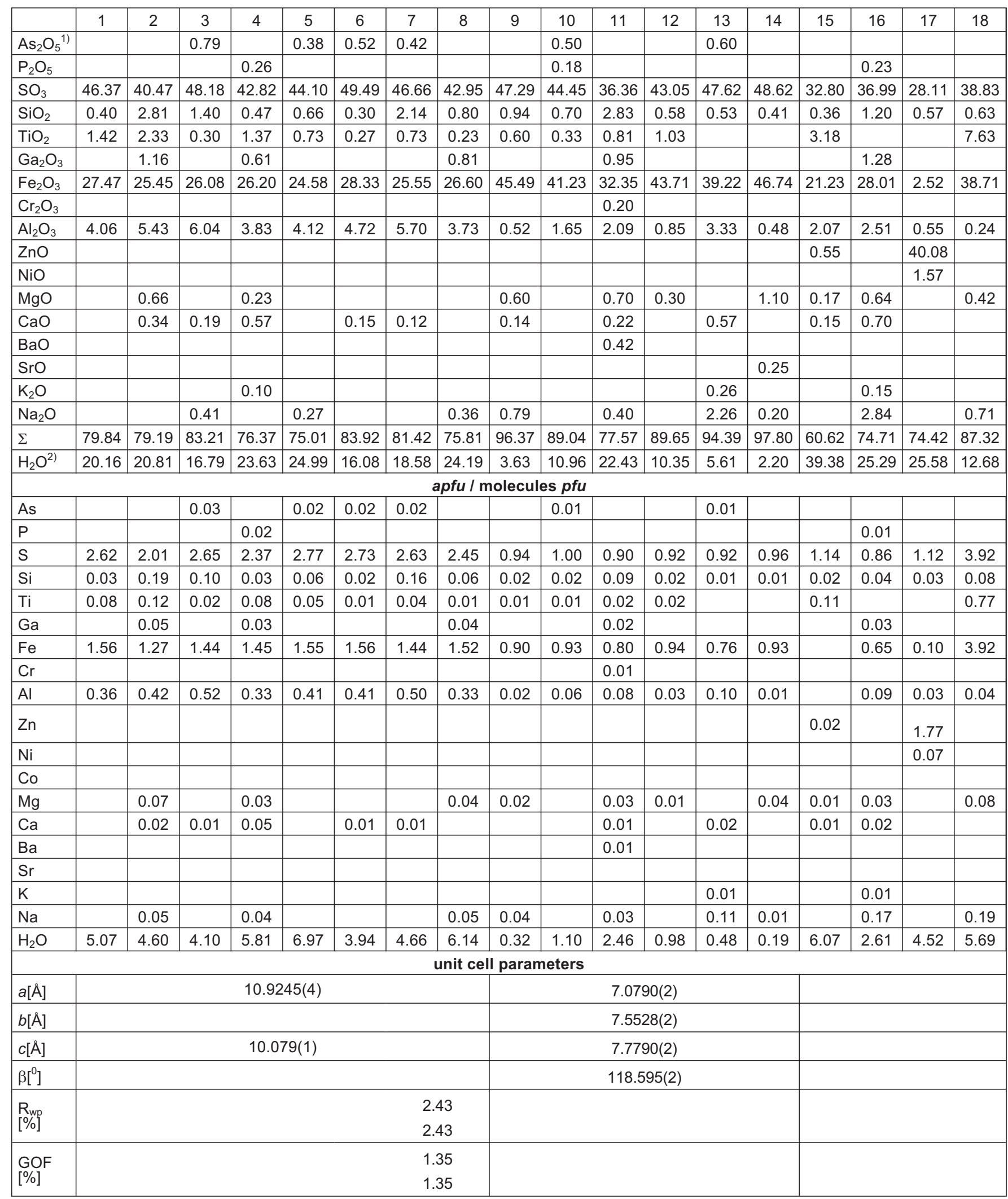

Analyses 1-8 - coquimbite; analyses 9-14 - szomolnokite; analysis 15 - ferrohexahydrite-like phase; analysis 16 - parabutlerite-like phase; analysis 17 - Zn-rich phase; analysis 18 - Ti-rich phase; ${ }^{1)}-$ Co was analysed but not observed; zero values are not reported; ${ }^{2)}-$ calculated as $^{2}$ $100-\Sigma$ 
Results of chemical analyses (in wt.\%) of sulphate minerals of sample Aou33

\begin{tabular}{|c|c|c|c|c|c|c|c|c|c|c|c|c|c|c|c|c|c|c|}
\hline & 1 & 2 & 3 & 4 & 5 & 6 & 7 & 8 & 9 & 10 & 11 & 12 & 13 & 14 & 15 & 16 & 17 & 18 \\
\hline $\mathrm{As}_{2} \mathrm{O}_{5}{ }^{1)}$ & & & & & & & & & & & & & 0.71 & & & & & \\
\hline $\mathrm{SO}_{3}$ & 30.91 & 26.76 & 29.68 & 35.38 & 33.61 & 33.46 & 37.04 & 42.02 & 41.11 & 30.42 & 25.44 & 23.01 & 32.81 & 33.44 & 35.72 & 31.48 & 32.78 & 31.42 \\
\hline $\mathrm{SiO}_{2}$ & & & & & & & & 0.16 & & & & & & 0.17 & & & & 0.12 \\
\hline $\mathrm{TiO}_{2}$ & & & & & & & & & & & & & & 0.17 & & & & \\
\hline $\mathrm{Ga}_{2} \mathrm{O}_{3}$ & 1.29 & 0.42 & 1.46 & 1.14 & 0.68 & 1.22 & 0.96 & 1.47 & 1.78 & 1.07 & 0.82 & 1.34 & & & & & & \\
\hline $\mathrm{Fe}_{2} \mathrm{O}_{3}$ & \begin{tabular}{|l|l|}
18.53 \\
\end{tabular} & \begin{tabular}{|l|}
17.72 \\
\end{tabular} & 18.03 & 23.05 & \begin{tabular}{|l|}
25.48 \\
\end{tabular} & \begin{tabular}{|l|}
26.06 \\
\end{tabular} & \begin{tabular}{|l}
25.79 \\
\end{tabular} & 27.29 & 26.47 & \begin{tabular}{|l|}
16.19 \\
\end{tabular} & 14.05 & 9.89 & 26.51 & 24.24 & 26.80 & 26.23 & \begin{tabular}{|l|}
26.51 \\
\end{tabular} & 28.09 \\
\hline $\mathrm{Al}_{2} \mathrm{O}_{3}$ & 2.02 & 2.02 & 1.64 & 1.35 & 2.14 & 1.74 & 2.30 & 0.69 & 1.24 & 0.75 & 2.19 & 3.69 & & & & & & \\
\hline $\mathrm{MnO}$ & & & & & & & & & & & 0.19 & & & & & & & \\
\hline $\mathrm{MgO}$ & & & & & & & & & & & & & 0.99 & 1.62 & 1.28 & 1.27 & 1.44 & 1.36 \\
\hline $\mathrm{CaO}$ & & & & & & & & & & & & & & 0.10 & & & 0.10 & \\
\hline $\mathrm{BaO}$ & & & 0.29 & & & & & & & & & & & & & & & 0.24 \\
\hline SrO & 0.29 & & 0.18 & & & 0.27 & & 0.23 & & & 0.27 & 0.23 & & & & & & \\
\hline $\mathrm{Na}_{2} \mathrm{O}^{2)}$ & 1.30 & 1.05 & 2.85 & 3.28 & 2.43 & 2.91 & 1.71 & 2.41 & \begin{tabular}{|l|}
3.82 \\
\end{tabular} & 2.22 & 1.62 & 1.62 & & & & & & \\
\hline$\Sigma$ & 54.45 & 48.17 & 54.13 & 64.20 & $\begin{array}{c}64.34 \\
34\end{array}$ & 65.65 & 67.80 & 74.27 & 74.42 & 50.75 & 44.69 & 39.92 & 61.02 & 59.74 & 63.80 & 59.05 & 60.83 & 61.23 \\
\hline $\mathrm{Na}_{2} \mathrm{O}^{3)}$ & 17.60 & 16.41 & 16.96 & 20.29 & 22.83 & 23.15 & 23.45 & 22.99 & 23.23 & 21.28 & 21.42 & 19.57 & & & & & & \\
\hline $\mathrm{H}_{2} \mathrm{O}^{4)}$ & 29.25 & 36.47 & 31.76 & \begin{tabular}{|l|}
18.79 \\
\end{tabular} & 15.26 & 14.10 & 10.46 & 5.15 & 6.17 & 30.19 & 35.51 & 42.13 & & & & & & \\
\hline $\mathrm{H}_{2} \mathrm{O}^{5)}$ & & & & & & & & & & & & & 38.98 & 40.62 & 36.20 & 40.95 & 39.17 & 38.77 \\
\hline \multicolumn{19}{|c|}{ apfu / molecules pfu } \\
\hline As & & & & & & & & & & & & & 0.06 & & & & & \\
\hline S & 1.35 & 1.26 & 1.36 & 1.35 & 1.14 & 1.12 & 1.22 & 1.41 & 1.37 & 1.66 & 1.38 & 1.37 & 5.91 & 5.96 & 6.00 & 1.09 & 1.11 & 1.01 \\
\hline $\mathrm{Si}$ & & & & & & & & & & & & & & 0.04 & & & & 0.01 \\
\hline $\mathrm{Ti}$ & & & & & & & & & & & & & & 0.03 & & & & \\
\hline $\mathrm{Ga}$ & 0.05 & 0.02 & 0.06 & 0.04 & 0.02 & 0.03 & 0.03 & 0.04 & 0.05 & 0.05 & 0.04 & 0.07 & & & & & & \\
\hline $\mathrm{Fe}$ & 0.81 & 0.83 & 0.83 & 0.88 & 0.87 & 0.87 & 0.85 & 0.92 & 0.88 & 0.89 & 0.76 & 0.59 & 4.79 & 4.33 & 4.51 & 0.91 & 0.90 & 0.91 \\
\hline $\mathrm{Al}$ & 0.14 & 0.15 & 0.12 & 0.08 & 0.11 & 0.09 & 0.12 & 0.04 & 0.06 & 0.06 & 0.19 & 0.34 & & & & & & \\
\hline $\mathrm{Mn}$ & & & & & & & & & & & 0.01 & & & & & & & \\
\hline $\mathrm{Mg}$ & & & & & & & & & & & & & 0.35 & 0.57 & 0.43 & 0.09 & 0.10 & 0.09 \\
\hline $\mathrm{Ca}$ & & & & & & & & & & & & & & 0.03 & & 6.29 & 5.88 & 5.56 \\
\hline $\mathrm{Ba}$ & & & 0.01 & & & & & & & & & & & & & & & \\
\hline $\mathrm{Sr}$ & 0.01 & & 0.01 & & & 0.01 & & 0.01 & & & 0.01 & 0.01 & & & & & & \\
\hline $\mathrm{Na}^{2)}$ & 0.15 & 0.13 & 0.34 & 0.32 & 0.21 & 0.25 & 0.15 & 0.21 & 0.33 & 0.31 & 0.23 & 0.25 & & & & & & \\
\hline $\mathrm{H}^{5)}$ & 11.37 & 15.22 & 18.62 & 6.37 & 4.60 & 4.19 & 3.07 & 1.54 & 1.83 & 14.64 & 17.12 & 22.21 & & & & & & \\
\hline $\mathrm{H}_{2} \mathrm{O}$ & & & & & & & & & & & & & & & & 6.29 & 5.88 & 5.56 \\
\hline \multicolumn{19}{|c|}{ unit cell parameters } \\
\hline$a[\AA]$ & \multicolumn{3}{|c|}{$7.275(3)$} & \multicolumn{6}{|c|}{$7.323(9)$} & \multicolumn{3}{|c|}{ 6) } & \multicolumn{3}{|c|}{$7.35(2)$} & \multicolumn{3}{|c|}{ 7) } \\
\hline$b[\AA \AA]$ & \multicolumn{3}{|c|}{$20.544(1)$} & \multicolumn{6}{|c|}{$16.06(7)$} & & & & \multicolumn{3}{|c|}{ 18.78(2) } & & & \\
\hline$c[\AA]$ & \multicolumn{3}{|c|}{$7.15(3)$} & \multicolumn{6}{|c|}{$7.10(2)$} & & & & \multicolumn{3}{|c|}{$7.32(2)$} & & & \\
\hline$\alpha\left[{ }^{0}\right]$ & & & & & & & & & & & & & \multicolumn{3}{|c|}{$90.60(2)$} & & & \\
\hline$\beta\left[^{0}\right]$ & & & & & & & & & & & & & \multicolumn{3}{|c|}{$102.25(2)$} & & & \\
\hline$\gamma\left[{ }^{0}\right]$ & & & & & & & & & & & & & \multicolumn{3}{|c|}{$99.08(13)$} & & & \\
\hline $\mathrm{R}_{\mathrm{wp}}$ & \multicolumn{18}{|c|}{17.86} \\
\hline GOF & \multicolumn{18}{|c|}{8.98} \\
\hline
\end{tabular}

Analyses 1-3 - sideronatrite; analyses 4-9 - metasideronatrite; analyses 10-12 - ferrinatrite; analyses 13-15 - copiapite group; analyses 16-18 - ferrohexahydrite-like phase; ${ }^{1)}-\mathrm{P}, \mathrm{Cr}$, Ni, Co and $\mathrm{K}$ were analysed but not observed; zero values are not shown; ${ }^{2)}-$ original and then recalculated values; ${ }^{3)}$ - calculated by stoichiometry [assuming apfu( $\left.\mathrm{Na}+\mathrm{Sr}+\mathrm{Ba}\right)=2$ ] ${ }^{4}$ ) - calculated backwards after recasting the cationic and anionic parts, then by difference (as $100-\Sigma) ;{ }^{5}$ total value, calculated backwards after charge-balance-based calculation of hydroxyl $\mathrm{H}$ assuming the ideal OH content of $1 \mathrm{pfu} ;{ }^{5)}$ - by difference $(100-\Sigma) ;{ }^{6)}$ - calculation impossible due to low content; ${ }^{7)}$ - calculation impossible as the analysed material is structurally different from the original one due to dehydration of the latter under the electron beam 
Results of chemical analyses (in wt.\%) of sulphate minerals of sample Aou35

\begin{tabular}{|c|c|c|c|c|c|c|c|c|c|c|c|c|c|c|}
\hline & 1 & 2 & 3 & 4 & 5 & 6 & 7 & 8 & 9 & 10 & 11 & 12 & 13 & 14 \\
\hline $\mathrm{As}_{2} \mathrm{O}_{5}{ }^{1)}$ & 1.30 & & 1.14 & \begin{tabular}{l|l|}
1.17 \\
\end{tabular} & & 1.41 & 1.20 & n.a. ${ }^{2)}$ & n.a. & & & 0.80 & n.a. & \\
\hline $\mathrm{SO}_{3}$ & \begin{tabular}{|l|}
45.07 \\
\end{tabular} & \begin{tabular}{|l|}
27.34 \\
\end{tabular} & 39.18 & \begin{tabular}{|l|}
43.48 \\
\end{tabular} & \begin{tabular}{|l|l}
41.41 \\
\end{tabular} & \begin{tabular}{|l|}
39.23 \\
\end{tabular} & \begin{tabular}{|l|}
40.32 \\
\end{tabular} & \begin{tabular}{|l|}
39.77 \\
\end{tabular} & & 34.73 & \begin{tabular}{|l|}
34.87 \\
\end{tabular} & \begin{tabular}{|l|}
31.04 \\
\end{tabular} & 28.06 & 30.71 \\
\hline $\mathrm{SeO}_{3}$ & & & & & & & & n.a. & n.a. & & & & n.a. & 0.57 \\
\hline $\mathrm{SiO}_{2}$ & 0.19 & 0.52 & 0.38 & 0.21 & 0.19 & 0.29 & & 0.38 & & & & & 1.78 & 0.45 \\
\hline $\mathrm{TiO}_{2}$ & & & & & & & 0.17 & n.a. & n.a. & 0.13 & & & & \\
\hline $\mathrm{Y}_{2} \mathrm{O}_{3}{ }^{3)}$ & & & & & & & & 0.85 & 1.33 & & & & & \\
\hline $\mathrm{La}_{2} \mathrm{O}_{3}$ & & & & & & & & 5.88 & 3.70 & & & & & \\
\hline $\mathrm{Ce}_{2} \mathrm{O}_{3}$ & & & & & & & & 17.83 & \begin{tabular}{|l|}
9.20 \\
\end{tabular} & & & & & \\
\hline $\mathrm{Pr}_{2} \mathrm{O}_{3}$ & & & & & & & & 3.10 & \begin{tabular}{|l|}
1.62 \\
\end{tabular} & & & & & \\
\hline $\mathrm{Nd}_{2} \mathrm{O}_{3}$ & & & & & & & & 7.62 & \begin{tabular}{|l|}
4.86 \\
\end{tabular} & & & & & \\
\hline $\mathrm{Sm}_{2} \mathrm{O}_{3}$ & & & & & & & & 1.11 & & & & & & \\
\hline $\mathrm{Gd}_{2} \mathrm{O}_{3}$ & & & & & & & & & 3.68 & & & & & \\
\hline $\mathrm{Ga}_{2} \mathrm{O}_{3}$ & & \begin{tabular}{|l|}
0.90 \\
\end{tabular} & & & & & & n.a. & n.a. & & & & 1.25 & \\
\hline $\mathrm{Fe}_{2} \mathrm{O}_{3}$ & 12.40 & \begin{tabular}{|l|}
13.46 \\
\end{tabular} & 21.00 & 9.67 & 10.70 & \begin{tabular}{|l|}
11.15 \\
\end{tabular} & \begin{tabular}{|l|}
12.36 \\
\end{tabular} & 5.48 & \begin{tabular}{|l|}
7.16 \\
\end{tabular} & 32.82 & \begin{tabular}{|l|}
32.76 \\
\end{tabular} & 29.81 & 24.54 & 4.84 \\
\hline $\mathrm{Cr}_{2} \mathrm{O}_{3}$ & 0.16 & & & & & & & n.a. & n.a. & 0.14 & & & & \\
\hline $\mathrm{Al}_{2} \mathrm{O}_{3}$ & \begin{tabular}{|l|}
10.27 \\
\end{tabular} & 2.47 & 6.68 & \begin{tabular}{|l|}
10.95 \\
\end{tabular} & 8.56 & 9.20 & 9.21 & 1.39 & \begin{tabular}{|l|}
0.42 \\
\end{tabular} & 1.37 & 1.28 & 1.43 & 1.89 & 0.34 \\
\hline $\mathrm{CuO}$ & & & & & & & & & & & & & & 0.41 \\
\hline $\mathrm{ZnO}$ & & & & & & & & & & & & & 0.52 & 42.52 \\
\hline $\mathrm{NiO}$ & & & & & & & & & & & & & 9.78 & 1.29 \\
\hline $\mathrm{MgO}$ & & 0.43 & & & & & & & & & & & 0.53 & \\
\hline $\mathrm{CaO}$ & & 0.57 & 0.16 & 0.13 & 0.33 & 0.21 & 0.12 & 8.97 & 8.85 & 0.35 & 0.42 & 0.29 & 0.43 & \\
\hline $\mathrm{SrO}$ & & 0.24 & & 0.19 & & & & 0.44 & n.a. & & & & & \\
\hline $\mathrm{Na}_{2} \mathrm{O}$ & & & & & & & & 1.03 & \begin{tabular}{|l|}
2.28 \\
\end{tabular} & & & & 1.01 & \\
\hline $\mathrm{K}_{2} \mathrm{O}$ & & \begin{tabular}{|l|}
0.07 \\
\end{tabular} & 0.08 & & & 0.10 & & & & & 0.08 & & & \\
\hline$\Sigma$ & 69.54 & 46.36 & 68.72 & 65.91 & 61.19 & 61.59 & 63.52 & 93.96 & 79.55 & 69.54 & 69.41 & 63.61 & 69.97 & 82.06 \\
\hline $\mathrm{H}_{2} \mathrm{O}^{4)}$ & \begin{tabular}{|l|}
30.46 \\
\end{tabular} & 53.64 & 31.28 & \begin{tabular}{|l|}
34.09 \\
\end{tabular} & 38.81 & \begin{tabular}{|l|}
38.41 \\
\end{tabular} & 36.48 & \begin{tabular}{|l|}
6.04 \\
\end{tabular} & 20.45 & 30.46 & 30.59 & \begin{tabular}{|l|}
36.39 \\
\end{tabular} & 30.03 & 17.94 \\
\hline \multicolumn{15}{|c|}{ apfu / molecules pfu } \\
\hline As & 0.06 & & 0.05 & 0.06 & & 0.08 & 0.06 & & & & & 0.02 & & \\
\hline S & 3.14 & 2.72 & 2.46 & 3.19 & 3.36 & 3.01 & 2.96 & 1.97 & 1.89 & 0.97 & 0.98 & 0.95 & 1.92 & 1.01 \\
\hline $\mathrm{Se}$ & & & & & & & & & & & & & & 0.01 \\
\hline $\mathrm{Si}$ & 0.02 & \begin{tabular}{|l|}
0.07 \\
\end{tabular} & 0.03 & 0.02 & 0.02 & 0.03 & & 0.03 & & & & & 0.16 & 0.02 \\
\hline $\mathrm{Ti}$ & & & & & & & 0.01 & & & & & & & \\
\hline$Y$ & & & & & & & & 0.03 & 0.05 & & & & & \\
\hline $\mathrm{La}$ & & & & & & & & 0.14 & 0.09 & & & & & \\
\hline $\mathrm{Ce}$ & & & & & & & & 0.43 & \begin{tabular}{|l|}
0.23 \\
\end{tabular} & & & & & \\
\hline $\mathrm{Pr}$ & & & & & & & & 0.07 & 0.04 & & & & & \\
\hline $\mathrm{Nd}$ & & & & & & & & 0.18 & 0.12 & & & & & \\
\hline $\mathrm{Sm}$ & & & & & & & & 0.03 & & & & & & \\
\hline $\mathrm{Gd}$ & & & & & & & & & 0.08 & & & & & \\
\hline $\mathrm{Ga}$ & & \begin{tabular}{|l|}
0.08 \\
\end{tabular} & & & & & & & & & & & 0.07 & \\
\hline $\mathrm{Fe}$ & 0.87 & 1.34 & 1.32 & 0.71 & 0.87 & 0.86 & 0.91 & 0.27 & 0.37 & 0.92 & 0.92 & 0.92 & 1.68 & 0.16 \\
\hline $\mathrm{Cr}$ & 0.01 & & & & & & & & & & & & & \\
\hline $\mathrm{Al}$ & 1.12 & 0.39 & 0.66 & 1.26 & 1.09 & 1.11 & 1.06 & 0.11 & 0.03 & 0.06 & 0.06 & 0.07 & 0.20 & 0.02 \\
\hline $\mathrm{Cu}$ & & & & & & & & & & & & & & 0.01 \\
\hline $\mathrm{Zn}$ & & & & & & & & & & & & & 0.03 & 1.76 \\
\hline $\mathrm{Ni}$ & & & & & & & & & & & & & 0.72 & 0.05 \\
\hline $\mathrm{Mg}$ & & & & & & & & & & & & & 0.07 & \\
\hline $\mathrm{Ca}$ & & 0.08 & 0.01 & 0.01 & 0.04 & 0.02 & 0.01 & 0.64 & 0.66 & 0.01 & 0.02 & 0.01 & 0.04 & \\
\hline $\mathrm{Sr}$ & & 0.02 & & 0.01 & & & & 0.02 & & & & & & \\
\hline $\mathrm{Na}$ & & & & & & & & 0.13 & 0.31 & & & & 0.18 & \\
\hline $\mathrm{K}$ & & 0.01 & 0.01 & & & \begin{tabular}{|l|}
0.01 \\
\end{tabular} & & & & & & & & \\
\hline $\mathrm{H}_{2} \mathrm{O}$ & 9.42 & 23.70 & 8.71 & 11.13 & 14.00 & 13.08 & 11.92 & 1.33 & 4.74 & 3.78 & 3.82 & 4.97 & 9.12 & 2.64 \\
\hline \multicolumn{15}{|c|}{ unit cell parameters } \\
\hline$a[\AA]$ & \multicolumn{6}{|c|}{$10.9199(7)$} & \multicolumn{3}{|c|}{ 5) } & & & & & \\
\hline$c[\AA]$ & \multicolumn{6}{|c|}{$17.070(2)$} & & & & & & & & \\
\hline $\mathrm{R}_{\mathrm{wp}}[\%]$ & \multicolumn{6}{|c|}{4.47} & & & & & & & & \\
\hline GOF [\%] & \multicolumn{6}{|c|}{1.35} & & & & & & & & \\
\hline
\end{tabular}


Table 7

Results of chemical analyses (in wt.\%) of sulphate minerals of sample Aou36

\begin{tabular}{|c|c|c|c|c|c|c|c|c|c|c|c|}
\hline & 1 & 2 & 3 & 4 & 5 & 6 & 7 & 8 & 9 & 10 & 11 \\
\hline $\mathrm{SO}_{3}{ }^{1)}$ & 34.79 & 31.78 & 35.35 & 33.94 & 33.05 & \begin{tabular}{|l|}
36.72 \\
\end{tabular} & 36.39 & \begin{tabular}{|l|}
33.87 \\
\end{tabular} & 26.19 & 27.74 & 33.66 \\
\hline $\mathrm{SeO}_{3}$ & & 0.52 & & & & & & & & & \\
\hline $\mathrm{La}_{2} \mathrm{O}_{3}{ }^{2)}$ & & & & & & & & & & & 5.22 \\
\hline $\mathrm{Ce}_{2} \mathrm{O}_{3}$ & & & & & & & & & & & 15.79 \\
\hline $\mathrm{Pr}_{2} \mathrm{O}_{3}$ & & & & & & & & & & & 2.05 \\
\hline $\mathrm{Nd}_{2} \mathrm{O}_{3}$ & & & & & & & & & & & 5.78 \\
\hline $\mathrm{Ga}_{2} \mathrm{O}_{3}$ & & & & & & & & & & & 1.07 \\
\hline $\mathrm{Fe}_{2} \mathrm{O}_{3}$ & 21.41 & 20.41 & 21.74 & 21.23 & 21.18 & 21.68 & 21.23 & 22.46 & 0.50 & 0.51 & 5.39 \\
\hline $\mathrm{Al}_{2} \mathrm{O}_{3}$ & 2.93 & 2.96 & 2.88 & 2.93 & 2.99 & 3.03 & 2.98 & 2.83 & & & 0.99 \\
\hline $\mathrm{MgO}$ & & & & & & & 0.67 & & & & \\
\hline $\mathrm{CaO}$ & & & 0.34 & 0.14 & 0.12 & 0.12 & 0.08 & 0.09 & & & 7.78 \\
\hline $\mathrm{BaO}$ & & & & & & & & & 46.51 & 46.73 & \\
\hline $\mathrm{SrO}$ & & & 0.30 & & & & 0.26 & & 3.22 & 3.37 & \\
\hline $\mathrm{Na}_{2} \mathrm{O}$ & & & & & & & & & 0.15 & 0.51 & \\
\hline $\mathrm{K}_{2} \mathrm{O}$ & & & & & & & & 0.08 & & & \\
\hline$\Sigma$ & 59.59 & 55.76 & 60.61 & 58.24 & 57.34 & 61.55 & 61.61 & 59.33 & 77.67 & 79.97 & 77.73 \\
\hline $\mathrm{H}_{2} \mathrm{O}^{3)}$ & 40.41 & 44.24 & 39.39 & 41.76 & 42.66 & 38.45 & 38.39 & 40.67 & & & 22.27 \\
\hline As & & & & & & & & & & & \\
\hline$S$ & 2.65 & 2.54 & 2.61 & 2.60 & 2.53 & 2.75 & 2.64 & 2.49 & 0.91 & 0.93 & 7.09 \\
\hline $\mathrm{Se}$ & & 0.03 & & & & & & & & & \\
\hline La & & & & & & & & & & & 0.54 \\
\hline $\mathrm{Ce}$ & & & & & & & & & & & 1.62 \\
\hline $\mathrm{Pr}$ & & & & & & & & & & & 0.21 \\
\hline $\mathrm{Nd}$ & & & & & & & & & & & 0.58 \\
\hline $\mathrm{Sm}$ & & & & & & & & & & & \\
\hline Gd & & & & & & & & & & & \\
\hline $\mathrm{Ga}$ & & & & & & & & & & & 0.19 \\
\hline $\mathrm{Fe}$ & 1.63 & 1.63 & 1.61 & 1.63 & 1.63 & 1.63 & 1.54 & 1.65 & 0.02 & 0.02 & 1.14 \\
\hline $\mathrm{Al}$ & 0.35 & 0.37 & 0.33 & 0.35 & 0.36 & 0.36 & 0.34 & 0.33 & & & 0.33 \\
\hline $\mathrm{Mg}$ & & & & & & & & & & & \\
\hline $\mathrm{aCa}$ & & & 0.04 & 0.02 & 0.01 & 0.01 & 0.01 & 0.01 & & & 2.34 \\
\hline $\mathrm{Ba}$ & & & & & & & & & 0.84 & 0.81 & \\
\hline $\mathrm{Sr}$ & & & 0.02 & & & & 0.01 & & 0.09 & 0.09 & \\
\hline $\mathrm{Na}$ & & & & & & & & & & & \\
\hline $\mathrm{K}$ & & & & & & & & 0.01 & & & \\
\hline $\mathrm{H}_{2} \mathrm{O}$ & 13.62 & 15.66 & 12.95 & 14.23 & 14.53 & 12.81 & 12.36 & 13.28 & & & 20.86 \\
\hline$a[\AA]]$ & \multicolumn{7}{|c|}{$10.909(2)$} & & & & \\
\hline$c[\AA]]$ & \multicolumn{7}{|c|}{$17.062(3)$} & & & & \\
\hline $\mathrm{R}_{\mathrm{wp}}[\%]$ & \multicolumn{7}{|c|}{11.45} & & & & \\
\hline GOF[\%] & \multicolumn{7}{|c|}{4.71} & & & & \\
\hline
\end{tabular}

Analyses 1-8-coquimbite grup, analyses 9,10 - baryte, analysis 11 - REE-rich phase; ${ }^{1)}-\mathrm{P}$, $\mathrm{Si}, \mathrm{Ti}, \mathrm{Cr}, \mathrm{Ni}, \mathrm{Co}, \mathrm{Cu}, \mathrm{Zn}$ and $\mathrm{As}$ were analysed but not observed; zero values are not shown; ${ }^{2)}$ REEs were measured in a non-standardized mode; ${ }^{3)}$ - by difference

Analyses 1-6 - aluminocoquimbite and coquimbite; analysis 7 - relatively pure aluminocoquimbite; analysis 8, 9 - REE-rich phase; analysis 10-12 - siderotil-like phase; analysis 13 - Ni(Zn)-rich phase; analysis $14-\mathrm{Zn}(\mathrm{Ni})$-rich phase; ${ }^{1)}$ - Ba was analysed but not observed; zero values are not shown; ${ }^{2)}$ - not analysed; ${ }^{3)}$ - REEs were measured in a non-standardized mode; ${ }^{4)}-$ by difference; ${ }^{5)}-$ impossible to calculate as the phase is derived from the original szomolnokite via its hydration [unit cell parameters of the latter are: $a=7.0674(8), b=7.558(1), c=$ 7.7676(8), $\beta=118.578(1) ; R_{\mathrm{wp}}$ and GOF as given above] 
example analyses in Table 5 with Nos. 16-18) may be $\left(\mathrm{Fe}^{2+}{ }_{0.70} \mathrm{Fe}^{3+}{ }_{0.19} \mathrm{Mg}_{0.08} \mathrm{Al}_{0.01}\right)_{\Sigma 0.98}\left(\mathrm{SO}_{4}\right)_{1.08} \cdot 6.10 \mathrm{H}_{2} \mathrm{O}$, somewhat fitting to ferrohexahydrite.

\section{SAMPLE Aou35}

The coquimbite group species of this sample fits to aluminocoquimbite. Sixteen analyses (with six examples in Table 6) can be recast to

$\left(\mathrm{Fe}_{0.69} \mathrm{Al}_{0.38} \mathrm{Ca}_{0.01}\right)_{\Sigma 1.08} \mathrm{Al}_{1.00}\left[\left(\mathrm{SO}_{4}\right)_{3.53}\left(\mathrm{AsO}_{4}\right)_{0.07}\right]_{\Sigma 3.10} \mathrm{Cl}_{0.01}$. . $x H 2 O)$, corresponding to $\mathrm{Acq}_{63} \mathrm{Dac}_{35} R_{2}$, where "Dac" corresponds to $\mathrm{AlAl}\left(\mathrm{SO}_{4}\right)_{3} \cdot 9 \mathrm{H}_{2} \mathrm{O}$ "dialuminocoquimbite" HEM, and " $R$ " to minor remaining As- and Ca-rich HEMs. A single analysis (No. 7 in Table 6) gives the following empirical formula: $\left(\mathrm{Fe}_{0.91} \mathrm{Al}_{0.06} \mathrm{Ca}_{0.01} \mathrm{Ga}_{0.01} \mathrm{Ti}_{0.01}\right)_{\Sigma 1.00} \mathrm{Al}_{1.00}\left[\left(\mathrm{SO}_{4}\right)_{2.96}\left(\mathrm{AsO}_{4}\right)_{0.06}\right]_{\Sigma 3.02}$. $11.92 \mathrm{H}_{2} \mathrm{O}$. This formula corresponds to a more pure aluminocoquimbite, with the mean end-members formula of $\mathrm{Acq}_{91} \mathrm{Dac}_{6} \mathrm{Gcq}_{1} \mathrm{Tcq}_{1} R_{1}$.

There are few interesting minor phases coexisting with aluminocoquimbite in sample Aou35. The first one, observed in at least two microareas as tiny BSE-bright microcrystals, may be given as $(\mathrm{Ca}, \mathrm{Na}, \mathrm{REE})_{2}(\mathrm{Fe}, \mathrm{REE})_{2}\left(\mathrm{SO}_{4}\right)_{4}(\mathrm{OH})_{2} \cdot x \mathrm{H} 2 \mathrm{O}$ (see analyses Nos. 8 and 9 in Table 6). Some of the REE are distributed to the $\mathrm{Fe}$ site, as a clear negative trend in the Fe-REE system is observed $\left(r^{2}=0.92\right)$. This phase may stand for a potentially new mineral species. The crystal chemistry of a second aluminocoquimbite associate may be given as

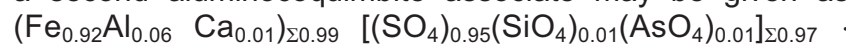
$5.19 \mathrm{H}_{2} \mathrm{O}(n=8)$, fitting to siderotil (ideally $\mathrm{FeSO}_{4} \cdot 5 \mathrm{H}_{2} \mathrm{O}$; analyses 10-12 in Table 6). Another single analysis corresponds to a Ni-rich $\mathrm{Zn}$-bearing phase (9.78 wt. \% NiO corresponding to 0.72 apfu Ni, analysis No. 13 in Table 6), possibly ideally $(\mathrm{Ni}, \mathrm{Zn})(\mathrm{Fe}, \mathrm{Al})_{2}\left(\mathrm{SO}_{4}\right)_{2}(\mathrm{OH})_{4} \cdot 7.5 \mathrm{H}_{2} \mathrm{O}$. The last single analysis (No. 14; Table 6) with 35.41 wt.\% ZnO (recalculated to 1.15 apfu $\mathrm{Zn}$ ) may correspond to a similar slightly Ni-enriched phase. The actual composition of this phase is unknown, as it is difficult to distinguish $\mathrm{Na}$ from $\mathrm{Zn}$ in EDS spectra. .

\section{SAMPLE Aou36}

The Aou36 coquimbite formula (normalized) is expressed as $\mathrm{Fe}_{1.00}\left(\mathrm{Fe}_{0.61} \mathrm{Al}_{0.38} \mathrm{Co}_{0.02} \mathrm{Ca}_{0.01} \mathrm{Mg}_{0.01}\right)_{\Sigma 1.03}\left[\left(\mathrm{SO}_{4}\right)_{2.99}\left(\mathrm{SeO}_{3}\right)_{0.01}\right]_{\Sigma 3.00}$. - $16.95 \mathrm{H}_{2} \mathrm{O}(n=16$, with 8 examples in Table 7). Baryte is an accessory mineral in the sample. It occurs as very tiny crystals. Its mean formula ( $n=2$, analyses Nos. 9 and 10 in Table 7) is $\left(\mathrm{Ba}_{0.87} \mathrm{Sr}_{0.09} \mathrm{Na}_{0.03} \mathrm{Fe}_{0.02}\right)_{\Sigma 1.01}\left(\mathrm{SO}_{4}\right)_{1.00}$. It relates to a proposed end-members formula of $\mathrm{Bar}_{86} \mathrm{Cel}_{9} \mathrm{Thn}_{3} \mathrm{FsO}_{2}$, where "Cel" stands for celestite, "Thn" for a thènardite $\left(\mathrm{Na}_{2} \mathrm{SO}_{4}\right)$ equivalent, and "Fso" for a hypothetical $\mathrm{FeSO}_{4}$ end-member. Another associate is a supposed $\mathrm{Ca}-\mathrm{REE}$ sulphate with a possible ideal formula of $\mathrm{Ca}_{2}[\mathrm{Ca}(\mathrm{Fe}, \mathrm{Al})](\mathrm{Ce}, \mathrm{Nd}, \mathrm{La}, \mathrm{Pr})_{3}\left(\mathrm{SO}_{4}\right)_{7}(\mathrm{OH})_{4} \cdot x \mathrm{H} 2 \mathrm{O}$ (analysis No. 11; Table 7). No analysable alunogen or szomolnokite crystals were found in the sample Aou36.

\section{CRYSTALLOGRAPHY}

The calculated unit cell parameters are juxtaposed with the literature-taken ones for the copiapite group (Fig. 4), coquimbite and aluminocoquimbite (Fig. 5), paracoquimbite (Fig. 6), alunogen (Fig. 7), the halotrichite group (Fig. 8), tamarugite (Fig. 9), melanterite (Fig. 10), and szomolnokite (Fig. 11). Span of the datapoints for the copiapite group is only slightly larger than the literature data, but it concerns all the six parameters. On the other hand, the difference for the cell-edge parameters is in the $0-0.87 \%$ range. The corresponding range for the angular parameters is $0.20-5.8 \%$; the larger discrepancy is explained below. Most of the datapoints fit to the copiapite/magnesiocopiapite field. The unit cell parameters calculated are especially close to that of Süsse (1972), Bayliss and Atencio (1985), RRUFF (Lafuente et al., 2015) and Kruszewski (2013). The unit cell parameter $c$ of the coquimbite group is largely invariable. Meanwhile, the parameter a has a moderately large spread (from $\sim 10.905$ to $10.97 \mathrm{C}$ ), the range being opened and finished with datapoints of the current study. Even those extreme values

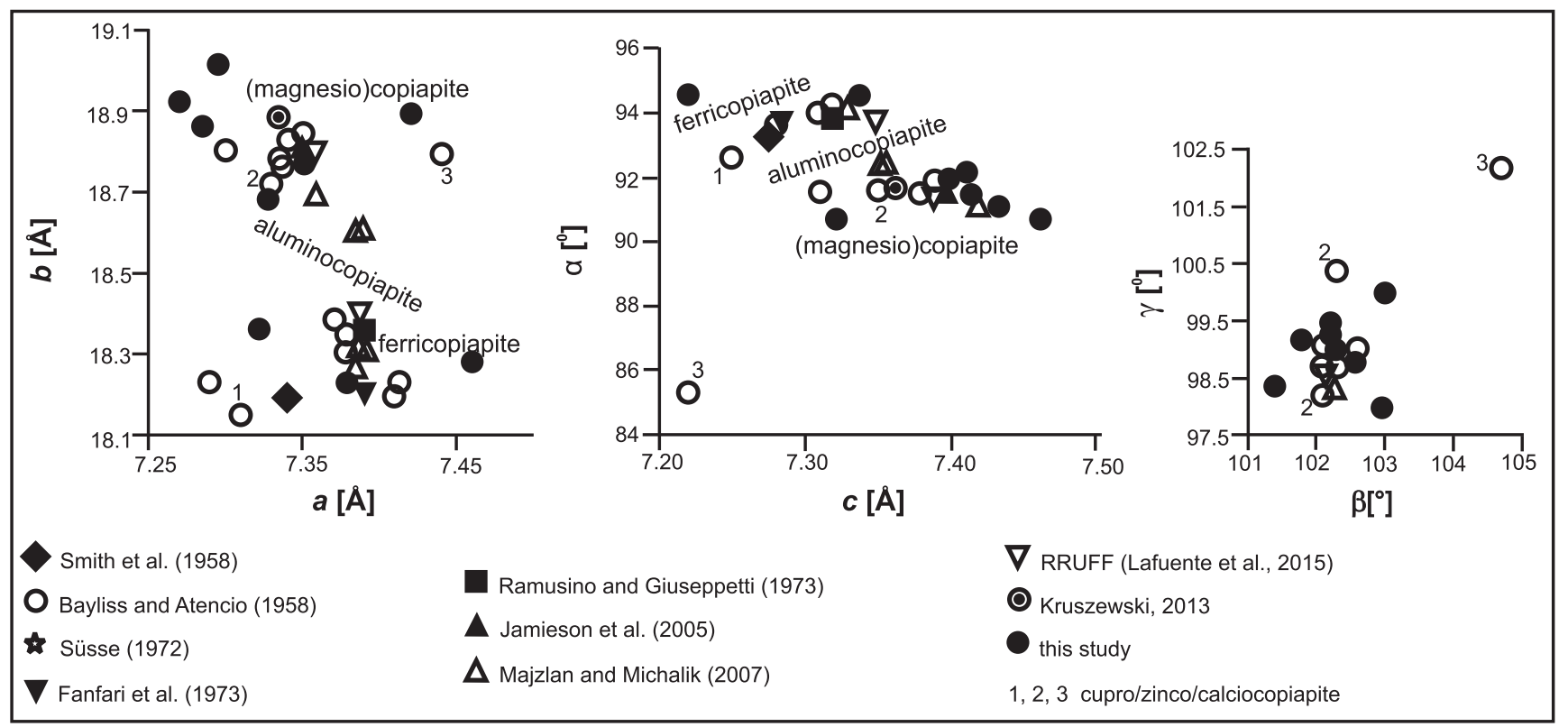

Fig. 4. Unit cell parameters for the Bhanine copiapite group members juxtaposed with literature data 


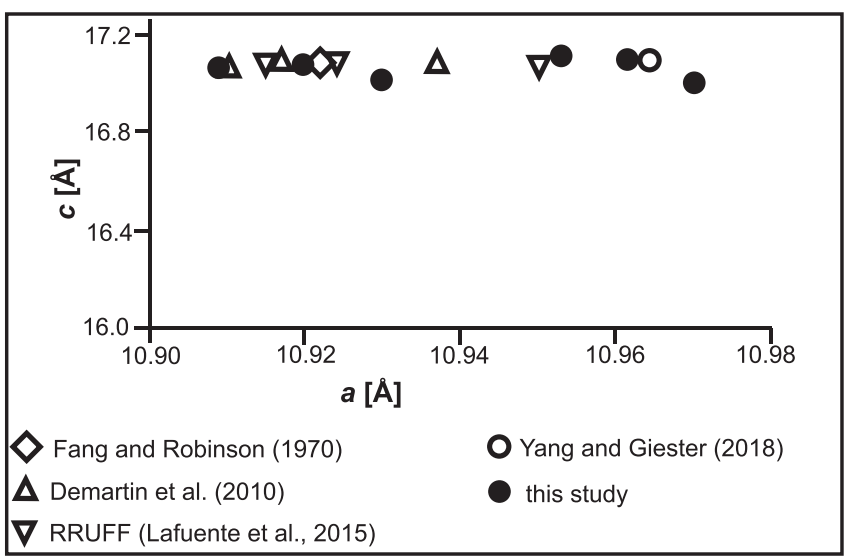

Fig. 5. Unit cell parameters for the Bhanine coquimbite group members juxtaposed with literature data

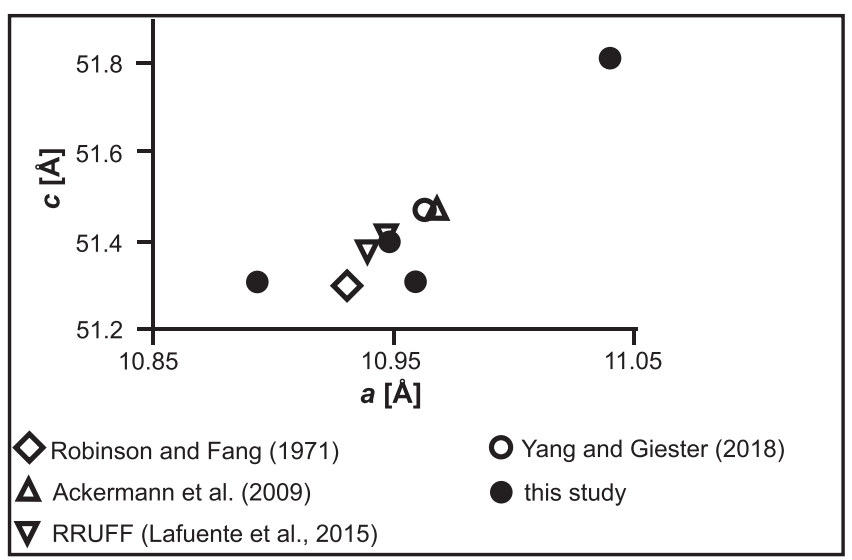

Fig. 6. Unit cell parameters for the Bhanine paracoquimbite juxtaposed with literature data

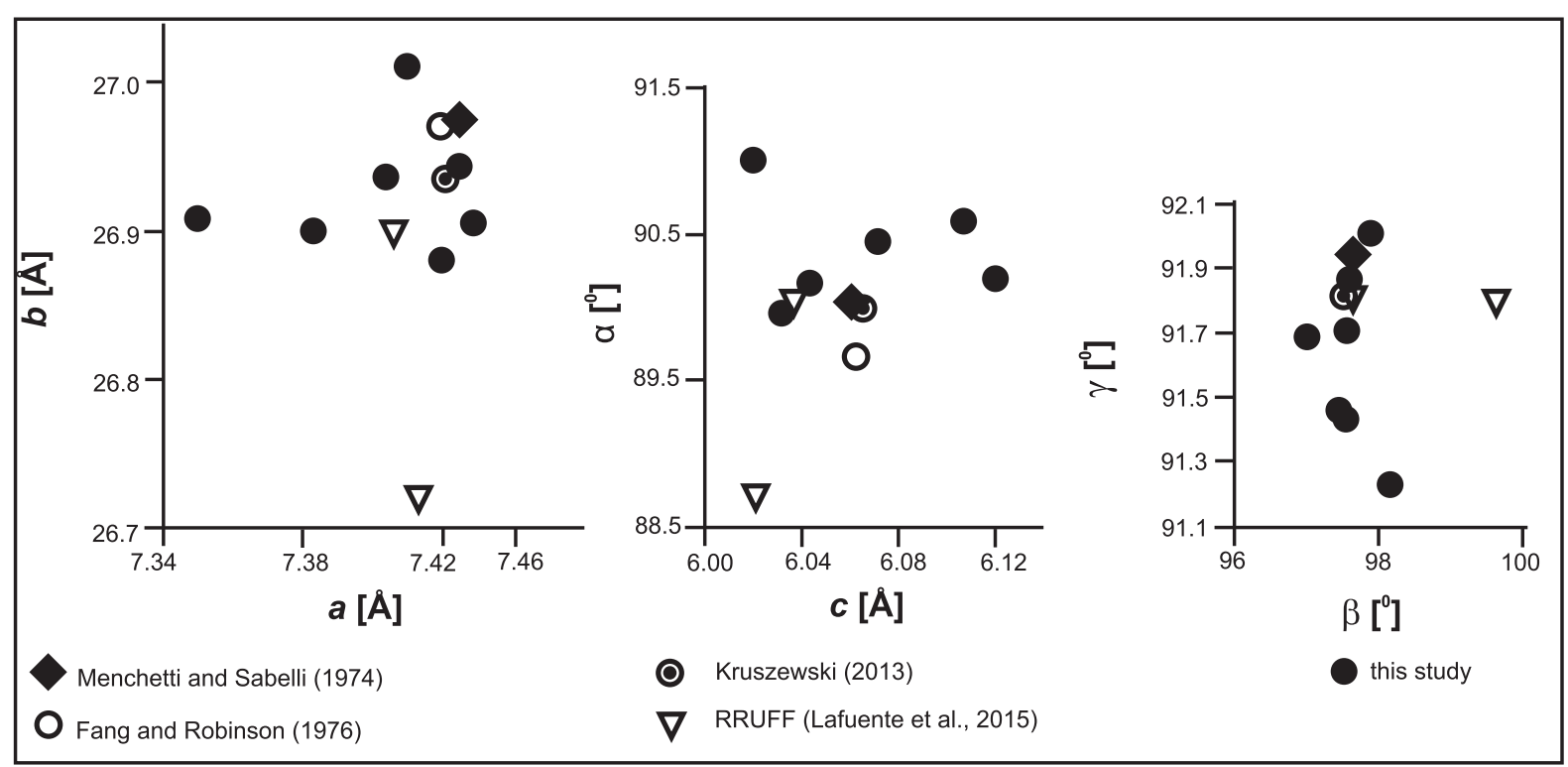

Fig. 7. Unit cell parameters for the Bhanine alunogen juxtaposed with literature data

are very close to the literature ones. Interestingly, the a values for aluminocoquimbite (from Demartin et al., 2010) are within the above range. Juxtaposing the a versus $c$ parameters of paracoquimbite shows a rather evident positive correlation and the margin values are, again, from the current study. Their divergence is, however, below the above percentage difference values. All the parameters calculated for alunogen also stand for threshold values, but the largest discrepancy is only slightly greater than $2 \%$. Many obtained values are similar to those of Menchetti and Sabelli (1974), Fang and Robinson (1976), Kruszewski (2013), and RRUFF (Lafuente et al., 2015). As opposed to most of the previous minerals, the unit cell parameters for halotrichite stay within the range delineated by the literature values. The Bhanine halotrichite parameters are especially similar to those of Lovas (1986), Majzlan et al. (2011), and RRUFF. A relatively clear positive trend is observed in the $a-c$ diagram. A similar situation concerns tamarugite: the unit cell parameters $a$ and $b$ plot in between the ones from Robinson and Fang (1969), Mereiter (2013), and ICDD PDF2005 database; the remaining parameters are close to those from Mereiter (2013) and RRUFF. The results obtained for melanterite are quite similar to those for pure melanterite of $\mathrm{Pe}$ terson (2003) and very similar to those from a very early study of Baur (1964). The unit cell parameters $a$ and $b$ of szomolnokite show a moderate spread, being somewhat similar to data of Wildner Giester (1961) and Majzlan et al. (2011). The remaining parameters are very similar to the ones of the first authors and to those taken from RRUFF.

No reliable fit could be obtained for sample Aou14 which seems to contain alum-(Na). The reflections of the latter strongly coincide with those ascribed to tamarugite. However, introduction of alum-(Na), alum-(K) or tschermigite to the model gives wrong results (e.g., $0.00 \mathrm{wt} \%$ of the alum). The unit cell parameters calculated for tamarugite of this sample are thus quite remarkable and somewhat unreliable (although standing within the range normally used as minimum/maximum con- 


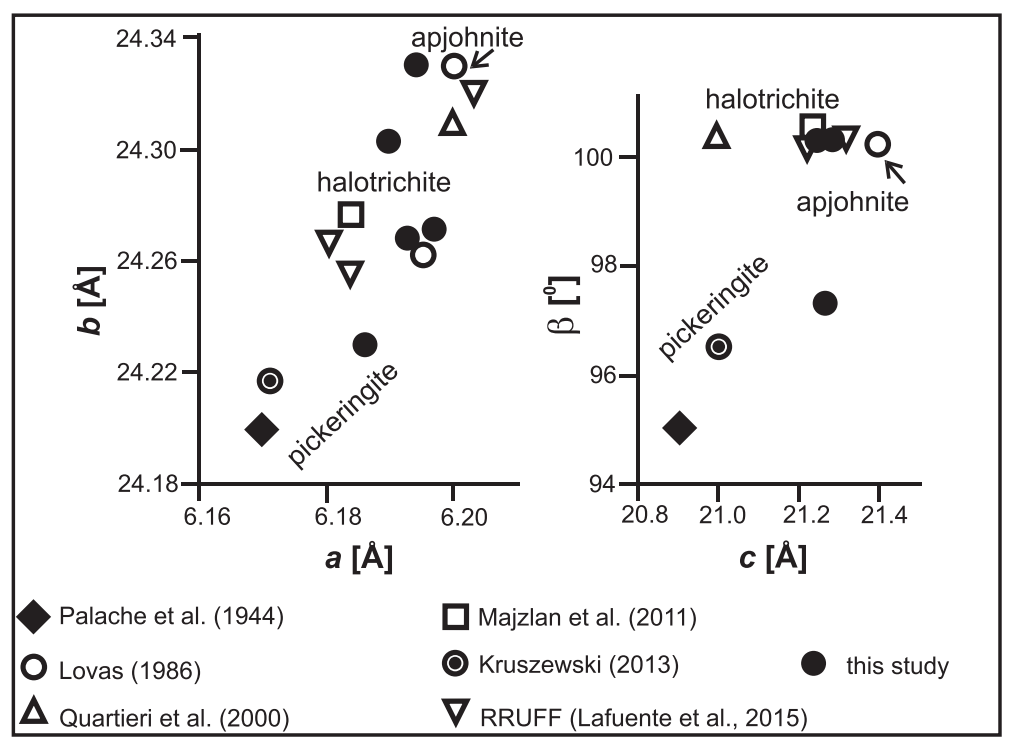

Fig. 8. Unit cell parameters for the Bhanine halotrichite group representatives juxtaposed with literature data

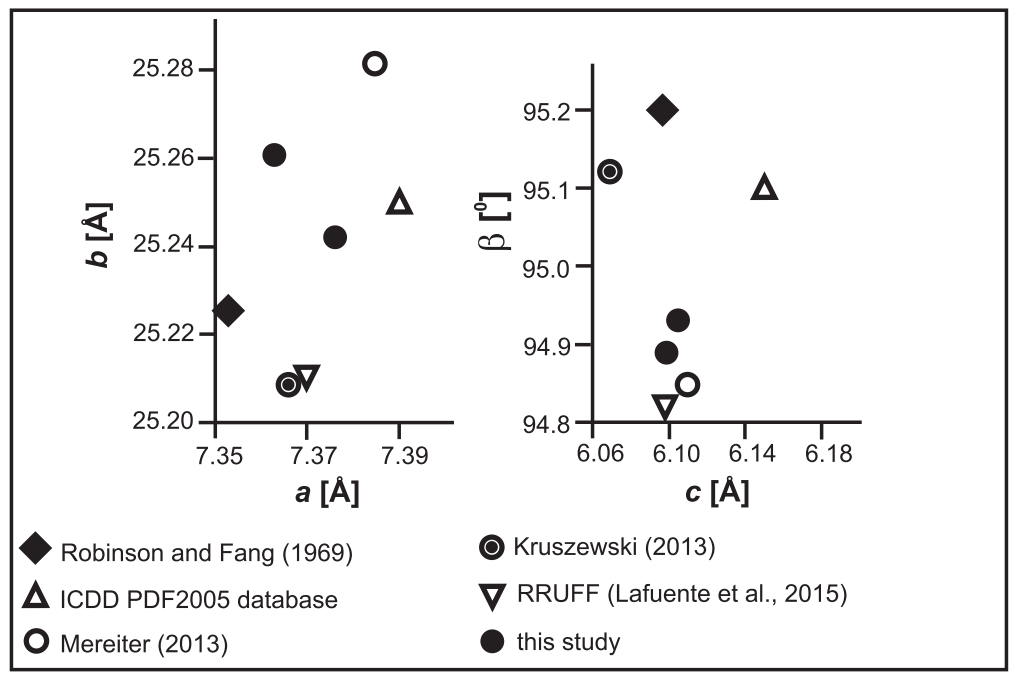

Fig. 9. Unit cell parameters for the Bhanine tamarugite juxtaposed with literature data

straints in TOPAS that is 99 and $101 \%$ of the initial parameters) as compared to the results for the other samples: $a=7.43(3), b$ $=25.46(4), c=6.04(3), \beta=96.15(3)$.

The greater variability of angles for many sulphate minerals, which are relatively low-symmetry crystals (i.e. triclinic or monoclinic), seems to be related to a larger number of degrees of freedom for the related atomic coordinates. The latter are considered to be resulting from a larger number of general instead of special positions for such lower-symmetry crystals (Pecharsky and Zavalij, 2003).

\section{GEOCHEMICAL REMARKS}

The sulphate mineral mixtures of the Bhanine coal occurrence usually do not pose an interesting geochemical issue from either sozological or industrial point of view. The maximum concentrations for selected geochemically meaningful trace elements (TEs) are: 58 ppm for V, 53 ppm for Cr, 200 ppm for Co, $280 \mathrm{ppm}$ for Ni, 300 ppm for $\mathrm{Cu}, 650$ ppm for Zn, 210 ppm for As, $55 \mathrm{ppm}$ for $\mathrm{Ba}, 3.3 \mathrm{ppm}$ for $\mathrm{Hg}, 30 \mathrm{ppm}$ for $\mathrm{Tl}$, and $26 \mathrm{ppm}$ for $\mathrm{Pb}$ (Table 8). Of these elements the only noteworthy ones are $\mathrm{Co}, \mathrm{Ni}, \mathrm{Cu}, \mathrm{As}, \mathrm{Hg}$ and $\mathrm{TI}$. The maximum enrichment factors of these elements as compared to Coal Clarkes (CC, see Table 9 for details) are 37, 16, 19, 23, 40 and 52, respectively, which may seem large. However, the noteworthy enrichment factors in regard to mean crustal abundances (MCAs) are large only in the case of As (up to 81 times), $\mathrm{Hg}$ (up to 40 times) and $\mathrm{TI}$ (up to 52 times), the values for other elements being lower or equal to 10. Sample Aou09 is the only one with noticeable Mn enrichment coinciding with its strongly ferrous character. Most of the maximum values concern the tamarugite-rich sample Aou22, which is also the only sample clearly enriched in cadmium (12 


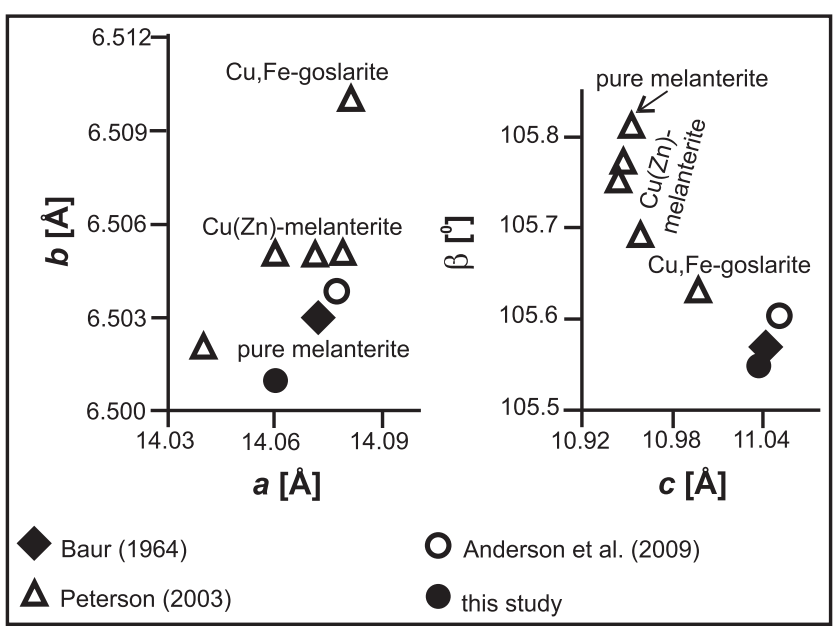

Fig. 10. Unit cell parameters for the Bhanine melanterite juxtaposed with literature data

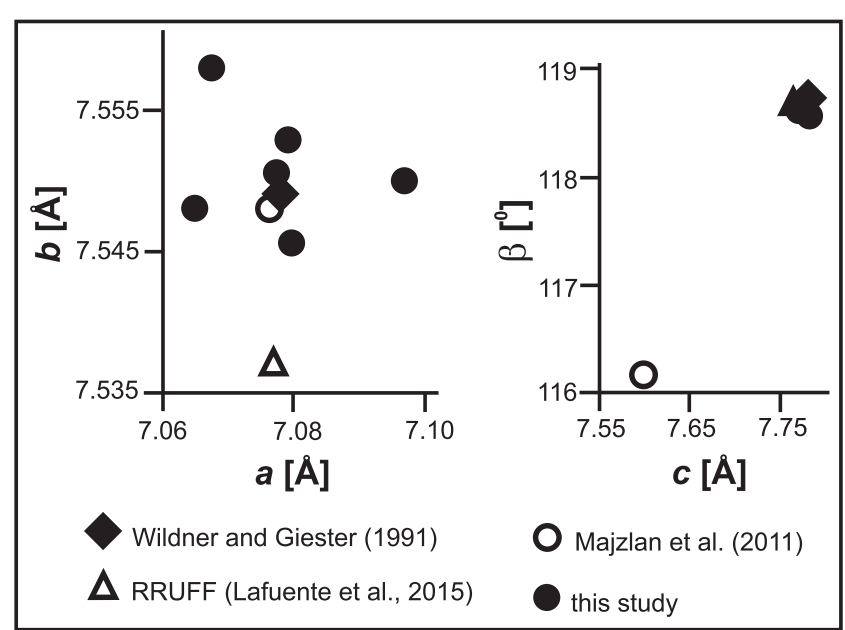

Fig. 11. Unit cell parameters for the Bhanine szomolnokite juxtaposed with literature data

Table 8

Geochemical analysis results for chosen sulphate mixture samples

\begin{tabular}{|c|c|c|c|c|c|c|c|c|c|}
\hline Sample & Aou09 & Aou11 & Aou22 & Aou27a & Aou29 & Aou32 & Aou33 & Aou35 & Aou $36 \mathrm{~b}$ \\
\hline \multicolumn{10}{|c|}{$\mathrm{ppm}$} \\
\hline $\mathrm{Si}$ & 0.00 & 0.00 & 0.00 & 0.00 & 0.00 & 0.00 & 0.00 & 0.00 & 0.00 \\
\hline $\mathrm{Ti}$ & 180 & 50 & 0.00 & 76 & 390 & 1.8 & 20 & 68 & 81 \\
\hline $\mathrm{V}$ & 19 & 58 & 0.00 & 21 & 29 & 0.00 & 2.7 & 29 & 11 \\
\hline $\mathrm{Cr}$ & 26 & 38 & 2.9 & 25 & 22 & 8.4 & 5.1 & 53 & 24 \\
\hline $\mathrm{Mn}$ & 1900 & 730 & 90 & 0.00 & 15 & 16 & 38 & 43 & 190 \\
\hline Co & 72 & 110 & 200 & 55 & 83 & 27 & 17 & 150 & 160 \\
\hline $\mathrm{Ni}$ & 190 & 140 & 280 & 170 & 160 & 44 & 17 & 190 & 170 \\
\hline $\mathrm{Cu}$ & 300 & 240 & 3.8 & 260 & 300 & 19 & 130 & 150 & 120 \\
\hline $\mathrm{Zn}$ & 160 & 45 & 440 & 30 & 74 & 97 & 24 & 420 & 650 \\
\hline $\mathrm{Ga}$ & 0.00 & 0.00 & 0.00 & 0.00 & 0.00 & 0.00 & 0.00 & 0.00 & 0.00 \\
\hline As & 100 & 93 & 200 & 64 & 93 & 36 & 37 & 170 & 210 \\
\hline $\mathrm{Se}$ & 0.00 & 0.00 & 0.00 & 0.00 & 0.00 & 0.00 & 0.00 & 0.00 & 0.00 \\
\hline $\mathrm{Sr}$ & $\sim 1000$ & $\sim 1000$ & 0.00 & $\sim 1000$ & $\sim 1000$ & 0.00 & $\sim 1000$ & $\sim 1000$ & 0.00 \\
\hline Mo & 0.00 & 0.00 & 0.00 & 0.00 & 0.00 & 0.00 & 0.00 & 0.00 & 0.00 \\
\hline $\mathrm{Cd}$ & 0.00 & 0.00 & 2 & 0.00 & 0.00 & 0.00 & 0.00 & 0.00 & 0.00 \\
\hline In & 0.00 & 0.00 & 6.5 & 0.00 & 0.00 & 0.00 & 0.00 & 0.00 & 0.00 \\
\hline $\mathrm{Sn}$ & 1 & 0.1 & 0.00 & 0.6 & 1 & 0.00 & 0.00 & 0.2 & 0.00 \\
\hline $\mathrm{Sb}$ & 0.00 & 0.00 & 2 & 0.00 & 0.00 & 0.00 & 0.00 & 0.00 & 0.6 \\
\hline $\mathrm{Ba}$ & 55 & 45 & 0.1 & 48 & 50 & 3.7 & 25 & 26 & 22 \\
\hline $\mathrm{Hg}$ & 0.9 & 2.3 & 3.3 & 1.5 & 1.9 & 0.5 & 0.2 & 2.4 & 4 \\
\hline $\mathrm{TI}$ & 30 & 24 & 0.00 & 30 & 25 & 1.4 & 15 & 14 & 9.3 \\
\hline $\mathrm{Pb}$ & 26 & 16 & 0.00 & 24 & 26 & 0.00 & 10 & 0.00 & 0.00 \\
\hline $\mathrm{Bi}$ & 0.00 & 0.00 & 0.00 & 0.00 & 0.00 & 0.00 & 0.00 & 0.00 & 0.00 \\
\hline \multicolumn{10}{|c|}{ wt. $\%$} \\
\hline $\mathrm{Mg}$ & 0.20 & 0.20 & 0.80 & 0.00 & 0.30 & 0.10 & 0.20 & 0.10 & 0.20 \\
\hline $\mathrm{Al}$ & 1.2 & 1.3 & 5.2 & 0.06 & 1.2 & 0.90 & 0.60 & 3.9 & 5.4 \\
\hline $\mathrm{Ca}$ & 0.10 & 0.30 & 0.20 & 0.30 & 0.10 & 0.00 & 0.60 & 0.10 & 0.10 \\
\hline $\mathrm{Fe}$ & 21 & 14 & 0.00 & 17 & 21 & 1 & 8.1 & 11 & 8.4 \\
\hline
\end{tabular}


Enrichment factors for trace elements of the sulphate samples as compared to the coal Clarke values (Ketris and Yudovich, 2009) and mean Earth's crust content (in parentheses: mean of 4 values, vide Parker, 1967, from crustal data of Vinogradov and Mason, continental-crustal data of Taylor, and igneous rocks data of Rankama)

\begin{tabular}{|l|c|c|c|c|c|c|c|c|c|}
\hline & Aou09 & Aou11 & Aou22 & Aou27a & Aou29 & Aou32 & Aou33 & Aou35 & Aou36b \\
\hline $\mathrm{Fe}$ & $(4)$ & $(3)$ & & $(3)$ & $(4)$ & & $(2)$ & $(2)$ & $(2)$ \\
\hline $\mathrm{V}$ & & 2 & & & & & & & \\
\hline $\mathrm{Cr}$ & & 2 & & & & & & & \\
\hline $\mathrm{Mn}$ & $27(2)$ & 10 & & & & & & & 3 \\
\hline $\mathrm{Co}$ & $12(3)$ & $18(5)$ & $37(10)$ & $9(3)$ & $14(4)$ & 5 & 3 & $25(7)$ & $27(7)$ \\
\hline $\mathrm{Ni}$ & $11(3)$ & $8(2)$ & $16(4)$ & $10(2)$ & $9(2)$ & 3 & & $11(3)$ & $10(2)$ \\
\hline $\mathrm{Cu}$ & $19(6)$ & $15(5)$ & & $16(5)$ & $19(6)$ & & $8(3)$ & $9(3)$ & $8(2)$ \\
\hline $\mathrm{Zn}$ & $6(2)$ & 2 & $15(5)$ & & 3 & 3 & & $15(5)$ & $23(8)$ \\
\hline $\mathrm{As}$ & $11(38)$ & $10(36)$ & $22(77)$ & $7(25)$ & $10(36)$ & $4(14)$ & $4(14)$ & $19(65)$ & $23(81)$ \\
\hline $\mathrm{Cd}$ & & & $10(12)$ & & & & & & \\
\hline $\mathrm{In}$ & & & $163(46)$ & & & & & & \\
\hline $\mathrm{Sb}$ & & & $2(4)$ & & & & & & \\
\hline $\mathrm{Hg}$ & $9(5)$ & $23(12)$ & $33(17)$ & $15(8)$ & $19(10)$ & $5(3)$ & 2 & $24(13)$ & $40(21)$ \\
\hline $\mathrm{Tl}$ & $52(32)$ & $41(26)$ & & $52(32)$ & $43(27)$ & 2 & $26(16)$ & $24(15)$ & $16(10)$ \\
\hline $\mathrm{Pb}$ & $3(2)$ & 2 & & $3(2)$ & $3(2)$ & & & & \\
\hline
\end{tabular}

times the MCA) and indium (163 times the CC), and showing some enrichment in antimony, too. The Cd,In,Sb- and other element enrichment in this sample correlates with its strongly aluminous character. Interestingly, the highest In enrichment in tamarugite (as opposed to other sulphate minerals) is also observed by Kruszewski (2013) for a coal-related sample from Poland.

\section{DISCUSSION}

The described sulphate assemblages are typical products of oxidation zones of pyrite-bearing mineralization (e.g. Buckby et al., 2003). Alunogen, for instance, is a common product of weathering of pyrite-bearing shales (Stracher et al., 2005). Fitzpatrick et al. (2010) identified sideronatrite-tamarugite-alunogen efflorescences in some Australian soils. They state that the first species is derived from acidic $(\mathrm{pH} 0.8-1.6)$ solutions arising from oxidation-dissolution of pyrite framboids. Where the $\mathrm{pH}$ is slightly higher (but still below 2.5), alunogen may be present. It should be noted that some sulphate minerals may arise due to oxidation coal-contained organic sulphur instead of pyrite. Such origin is suggested for coquimbite, jarosite, alunogen, and other sulphates from Bukit Asam (Sumatra, Indonesia; Susilawati, and Ward, 2006).

Both the pyrite oxidation and the sequence of crystallisation of $\mathrm{Fe}$ sulphates and their mutual interactions are relatively well-known. The first phenomena may be written as two basic reactions (e.g., Dill et al., 2002):

(1) $\mathrm{FeS}_{2}+7 \mathrm{O}_{2}+8 \mathrm{H}_{2} \mathrm{O} \rightarrow \mathrm{FeSO}_{4} \cdot 7 \mathrm{H}_{2} \mathrm{O}+\mathrm{SO}_{4}^{2-}+2 \mathrm{H}^{+}$

(2) $\mathrm{Fe}^{2+}+0.25 \mathrm{O}_{2}+\mathrm{H}^{+} \rightarrow \mathrm{Fe}^{3+}+\mathrm{H}_{2} \mathrm{O}$

The above reactions lead to the formation of highly acidic solutions rich in ferrous, ferric and sulphate ions; their direct crystallisation in the presence of large amounts of water leads to formation of copiapite and coquimbite, according to reactions (3) and (4), respectively (Hudson-Edwards et al., 1999):

(4) $\mathrm{Fe}^{2+}+4 \mathrm{Fe}^{3+}+6 \mathrm{SO}_{4}^{2-}+22 \mathrm{H}_{2} \mathrm{O} \rightarrow \mathrm{FeFe}_{4}\left(\mathrm{SO}_{4}\right)_{6}(\mathrm{OH})_{2}$ $2 \mathrm{H}_{2} \mathrm{O}+2 \mathrm{H}^{+}$
(5) $2 \mathrm{Fe}^{3+}+3 \mathrm{SO}_{4}^{2-}+9 \mathrm{H}_{2} \mathrm{O} \rightarrow \mathrm{Fe}_{2}\left(\mathrm{SO}_{4}\right)_{3} \cdot 9 \mathrm{H}_{2} \mathrm{O}$

The above reactions and oxidative interaction may explain coexistence of minerals of the coquimbite and copiapite groups in the Bhanine samples, considering that the aforementioned solutions extract some metals $(\mathrm{K}, \mathrm{Na}, \mathrm{Ca}, \mathrm{Mg}, \mathrm{Al})$ from the surrounding rocks.

The oxidative coquimbite-copiapite relation noticed by Hudson-Edwards et al. (1999) does not apply to two samples where the $\mathrm{Fe}^{2+}$-dominant copiapite group is present (samples Aou20 and Aou33), as these samples are devoid of coquimbite. It is, however, suggested that copiapite in the strict sense might existed in many samples and was oxidized to aluminous coquimbite and aluminocoquimbite by $\mathrm{Fe}^{3+}$-bearing acidic solutions also rich in Al. Such phenomenon would explain (1) the Al-dominant composition of many copiapites analysed, (2) important crystallochemical role of Al in the coquimbite group, and (3) Al entering, in small amounts, the nominally non-aluminous minerals like Fe(II) sulphates. Another explanation is the simultaneous crystallisation of Al-dominant end-members of the copiapite and coquimbite groups. Changing $\mathrm{Al}^{3+}$ activity might drive the prevalence of these end-members in the composition of the particular crystals.

The Bhanine sulphate assemblages are largely $\mathrm{Fe}-$ and Al-dominant, with addition of $\mathrm{Mg}, \mathrm{Ca}$ and $\mathrm{Na}$ and only minor amounts of $\mathrm{K}$. Thus, the source of the non-iron cations must rather be Al-rich. It is expected that this role is played by clay minerals. Indeed, kaolinite is the main clay mineral present as an impurity in some of the samples studied. Meanwhile, $\mathrm{Ca}, \mathrm{Na}$ and $\mathrm{Mg}$ are possibly derived from either basaltic or tuffaceous rocks associated with the Chouf sandstone. The carbonate source of $\mathrm{Ca}$ and $\mathrm{Mg}$ seems somewhat unlikely, as carbonate rocks are not reported from the area. An important additional source of $\mathrm{Na}$ may be the sea breeze; indeed, occurrences of tamarugite in some near-shore areas are known (e.g., Segnit, 1976).

From Figures 2 and 3 it can easily be remarked that halotrichite is a late phase, postdating either $\mathrm{Fe}^{3+}$-rich sulphates (copiapite group, coquimbite group) and Al sulphates (alunogen). Such its location in the crystallisation sequence fits to a crystallisation path reported by O'Connor (2005) for sulphate 
assemblages of Iron Mountain, Australia, which begins from crystallisation of melanterite, runs through rozenite, szomolnokite, copiapite, römerite, coquimbite, kornelite, rhomboclase and voltaite up to halotrichite-bílinite. The author also states that szomolnokite may be the last species in the crystallisation sequence of Fe sulphates, but it may also be transformed to (1) copiapite, römerite and bílinite, or (2) rhomboclase and later voltaite, depending on the rate of dehydration, activity of some ions (e.g., $\mathrm{K}^{+}$), and variation of the local humidity. The melanterite-siderotil-rozenite-szomolnokite path fits the observations of Montano (1981), who found szomolnokite as the prevailing sulphate identified in some coal samples, the mineral being followed by rozenite and melanterite. As seen in Figure $2 \mathrm{E}$, tamarugite seems to be formed synchronically to jarosite. Based on the macro- and microscopic observations, copiapite-group minerals seem to be the first species formed. Gypsum can either occur as a late phase (overgrowing minerals of the copiapite and coquimbite groups, as in Fig. $2 \mathrm{~A}$ ), or predate $\mathrm{Fe}$ sulphates (jarosite-metasideronatrite mixture; Fig. 2F). The curved-crust habit of the sulphate minerals is relatively well-known and may be related to a two stage-process: (1) capillary infiltration of (rain) water, and (2) capillary recurrence of water, followed by solution evaporation (e.g., Fernández-Remolar et al., 2005; Velasco et al., 2005). The first authors also point to the curved shape of sulphate aggregates, suggesting it to arise from the interaction of basement (ir)regularities and seasonal exposition. Socket-like aggregates are also reported by Yalovik et al. (2016), who suggest them to result from pseudomorphism.

The sulphate minerals analysed do not seem to be very outstanding in terms of their major-element chemistry. There are, however, some geochemical phenomena to be discussed. Few samples studied contain some trace minerals that might possibly suggest fire phenomena to occur in Lebanon. Rostite, likely present in sample Aou01 and possibly in Aou17, Aou24 and Aou36, is a mineral typical for coal fires (e.g., Čech, 1979; Kruszewski et al., 2018a). It should, however, be noted that single occurrences of rostite in oxidation zones of some ore deposits are also known (e.g., Skarpelis and Argyraki, 2009). Another mineral often found associated with coal fires is mikasaite (possibly present in sample Aou31); occurrences of such kind are described, e.g. by Miura et al. (1994). Trace native sulphur is present in sample Aou34 and this mineral is even more common in burning coal-mining heaps (e.g., Kruszewski et al., 2018a). Native sulphur may, however, be also biogenic (e.g., Lutz Ehrlich and Newman, 2009). the assemblages studied are devoid of the best coal-fire fingerprint mineral, that is salammoniac.

Due to the intimate association of the sulphate minerals and their intergrowths with non-sulphate ones containing $\mathrm{Si}, \mathrm{K}$, and probably $\mathrm{Na}$ and $\mathrm{Ca}$, the reported empirical formulas should be treated carefully. However, $\mathrm{K}, \mathrm{Na}$ and $\mathrm{Ca}$ may enter the otherwise $\mathrm{Fe}-$, $\mathrm{Al}$ - or Mg-dominant sites at least in some minerals. This has especially to do with the copiapite group (e.g., Jamieson et al., 2005). All the above alkaline metals are reported in aluminocopiapite analyses of Berry (1947), with apfu Ca included in the empirical formula. Cobalt and copper are also known to be able to enter copiapites, as shown for Sydney Coalfield (Nova Scotia, Canada) magnesiocopiapite with 0.16 wt.\% $\mathrm{CoO}$ and 0.05 wt.\% CuO (Zodrow, 1980). Possible occurrence of trace $\mathrm{Ca}$ in the $\mathrm{ASO}_{4}$-stoichiometry Mg sulphates is reported by Baur and Rolin (1972). As such sulphates are structurally similar to simple Fe sulphates, trace Ca could also be expected present in the latter. A supposed unnamed Al-analogue of melanterite is reported by O'Connor (2005). Titanium substitution in many of the minerals studied is not surprising if one compares it with the sulphate assemblages known from Chile. Such assemblages are often rich in minerals of the copiapite group (Rosse, 1883) and tamarugite (Bandy, 1938), and two minerals with $\mathrm{Ti}$ as a species-defining element were discovered quite recently (alcaparrosaite, Kampf et al., 2012; calamaite, Pekov et al., 2016).

One particular element probably highly enriched in the sulphates analysed is gallium. Gallium is known from its affinity to coals and a halogenide mineral having $>3 \mathrm{wt} . \% \mathrm{Ga}_{2} \mathrm{O}_{3}$ is described by Kruszewski et al. (2006; 2018b) from a coal-rich environment. However, this element content needs approval using other methods like WDS. The measured concentrations of As and $\mathrm{Cu}$ are somewhat comparable to those reported by Jamieson et al. (2005; Table 3) for copiapites from the Richmond mine (190 ppm As, 550 ppm Cu), but the Bhanine samples are depleted in Zn as compared to 1870 ppm Zn from their study. Most of the Bhanine TEs concentrations ( $\mathrm{V}$ and Ni being exceptions) are also way inferior to the maximum AMD-tailing values reported by Bobos et al. (2006): 1618 ppm As, 1073 ppm $\mathrm{Cu}, 1050$ ppm Ba, 157 ppm Co, 95 ppm Cr, and 10955 ppm Pb. These tailings (of Aljustrel, Portugal) are composed of siderotil, copiapite, rhomboclase, natrojarosite, gypsum and some amorphous $\mathrm{Fe}-\mathrm{O}-\mathrm{OH}$ compounds. The corresponding maximum values reported by Buckby et al. (2003) for river-bank evaporates from Río Tinto, Spain, are: 16500 ppm Cu and 558 ppm Co (in melanterite), 518 ppm Ni (in copiapite), 1210 ppm Pb (in gypsum), 49 ppm V (in szomolnokite-halotrichite-copiapite mixture), and 27100 ppm Zn (in copiapite-szomolnokite-halotrichite mixture). Thus, the Bhanine sulphate mineral mixtures as compared to the above examples do not pose a serious environmental issue.

As stated in the previous chapter, the most evident enrichment of the Bhanine sulphates in TEs concerns $\mathrm{Co}, \mathrm{Ni}, \mathrm{Cu}, \mathrm{As}$, $\mathrm{Hg}$ and $\mathrm{TI}$. The Coal Affinity Indexes, being the measures of the elements affinities to the coaly matter, reported for these elements, are 3.0, 2.1, 2.3, 6.2, 11, and 5.5, respectively (Ketris, Yudovich, 2009). Meanwhile, Parzentny, Lewińska-Preis (2006) point to an important role of coal-disseminated sulfide minerals in concentration of chalcophile elements like $\mathrm{Cd}$, Co, $\mathrm{Cu}, \mathrm{Ni}, \mathrm{Pb}$, and $\mathrm{Zn}$. Coal-contained pyrite may also be an essential source of As (e.g., Kruszewski et al., 2018a). It is thus suggested that the Bhanine coal was the source of $\mathrm{Hg}$ and $\mathrm{TI}$, while $\mathrm{Co}, \mathrm{Ni}$ and $\mathrm{Cu}$ were derived from the local pyrite, and $\mathrm{As}$ came from both those sources. However, to check these assumptions and to point to main mineral sinks of the particular TEs, simple element-to-element content diagrams were constructed (Fig. 12). The particular TEs were found to be correlated with:

- aluminum, being positively correlated with zinc $\left(r^{2}=0.93\right)$, arsenic $\left(r^{2}=0.92\right)$, cobalt $\left(r^{2}=0.82\right)$, and mercury $\left(r^{2}=0.73\right)$;

- arsenic, positively correlated with zinc $\left(r^{2}=0.87\right)$, mercury $\left(r^{2}=0.84\right)$, and to some extent with nickel $\left(r^{2}=0.59\right)$;

- cobalt, positively correlated with arsenic $\left(r^{2}=0.90\right)$, mercury $\left(r^{2}=0.82\right)$, and nickel $\left(r^{2}=0.69\right)$; strongly negatively correlated with thallium $\left(r^{2}=0.97\right)$, when discluding samples Aou32 and Aou33;

- iron, strongly positively correlated with copper $\left(r^{2}=0.98\right)$, lead $\left(r^{2}=0.95\right)$, barium $\left(r^{2}=0.96\right)$ and thallium $\left(r^{2}=0.91\right)$;

- lead, positively correlated with barium $\left(r^{2}=0.85\right)$ but negatively with calcium $\left(r^{2}=0.84\right)$

- nickel, showing very strong correlation with barium $\left(r^{2}=0.99\right)$;

- thallium, showing strong positive correlation with barium $\left(\mathrm{r}^{2}\right.$ $=0.97)$ and vanadium $\left(r^{2}=0.70\right)$, the latter being somewhat positively correlated with chromium $\left(r^{2}=0.58\right)$; 

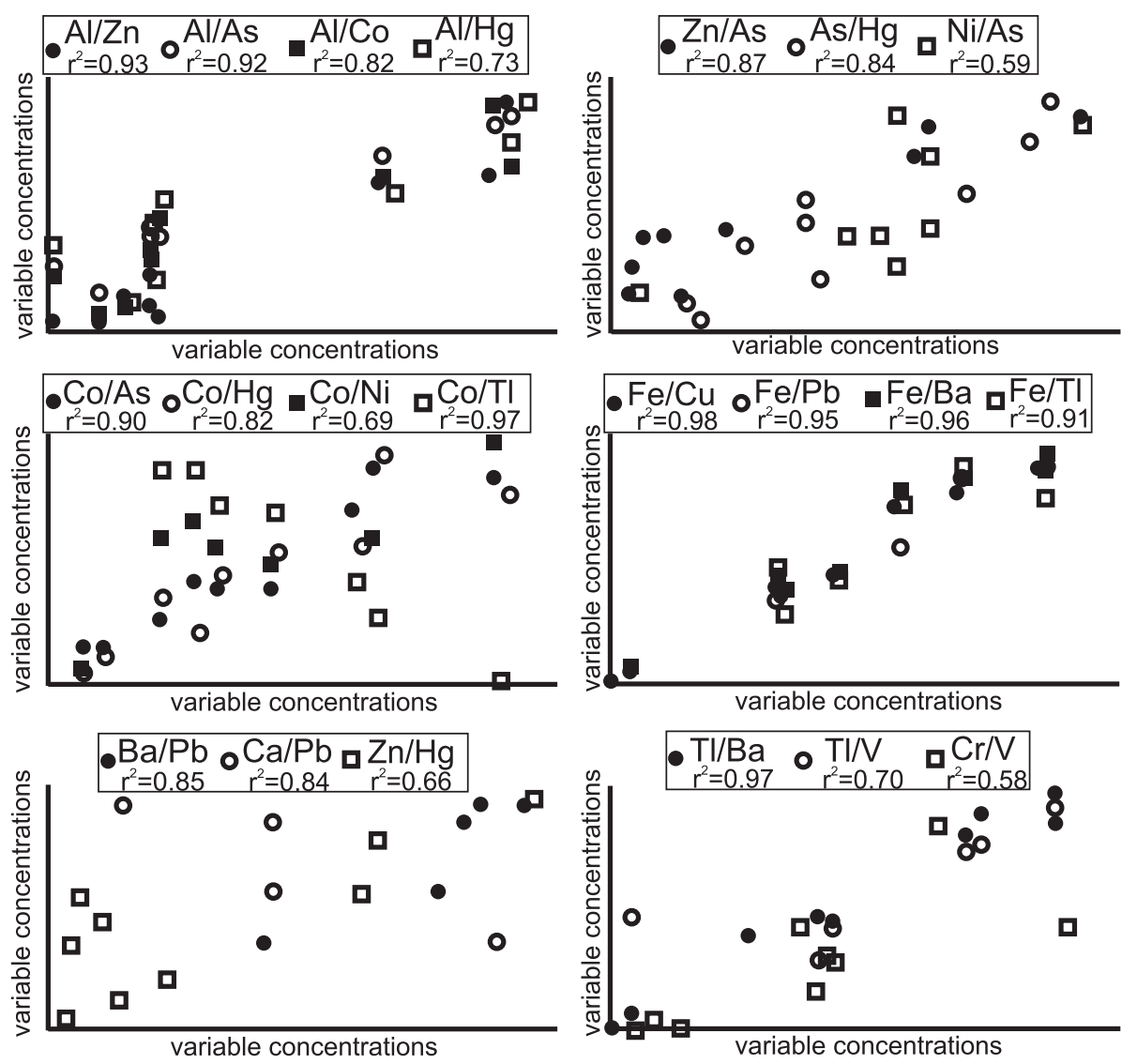

Fig. 12. Trace element ratio diagrams

Zero values were omitted in case of $\mathrm{Ba} / \mathrm{Pb}, \mathrm{Ca} / \mathrm{Pb}$ and $\mathrm{TI} / \mathrm{V}$ ratios

- zinc, positively correlated with mercury $\left(r^{2}=0.66\right)$.

The above $r^{2}$ values are based on non-zero datapoints in case of the $\mathrm{Ba} / \mathrm{Pb}, \mathrm{Ca} / \mathrm{Pb}$ and $\mathrm{TI} / \mathrm{V}$ relations. The above coefficients suggest the existence of some groups of coexisting elements:

- Al-Co-Ni-Zn-Hg-As,

- Fe-Pb-Ba-Cu-Tl,

$-\mathrm{V}-\mathrm{Cr}$.

It thus seems like cobalt and nickel, as opposed to their common geochemical affinity to iron (Goldschmidt, 1937), are not behaving here as siderophiles. It is also this group which preferentially concentrates arsenic and mercury. As opposed to that, copper, lead, barium and thallium seem to be concentrated in Fe-rich sulphates.

\section{CONCLUSIONS}

Oxidation of pyrite in the coal-bearing sandpit in the Bhanine Valley of south Lebanon has led to the formation of abundant sulphate mineral mixtures. The mixtures are highly dominated by Fe- and Al-rich sulphates. Of the first group, both $\mathrm{Fe}$ (II) sulphates (the most common szomolnokite, less common rozenite, and subordinate melanterite) and $\mathrm{Fe}(\mathrm{III})$ sulphates (coquimbite, aluminocoquimbite, paracoquimbite, aluminocopiapite, copiapite, and some minor species) are present. The Al sulphates are mainly alunogen and tamarugite, but the highly aluminous character of both copiapite and coquimbite groups is evident. The latter also concerns the nominally Al-free $\mathrm{Fe}$ (II) sulphates. Aluminium is dominant in the composition of many minerals analysed, including the coexisting copiapite and coquimbite groups. It is thus suggested that these Al-rich minerals may have formed in transformation of original $\mathrm{Fe}^{2+}$-rich materials by action of $\mathrm{Fe}^{3+}$ - and Al-bearing solutions. On the other hand, changes in $\mathrm{Al}^{3+}$ activity in the solutions may simply allow Al to enter and modify the structures of the primary sulphate species. The Bhanine sulphate mixtures are not important collectors of TEs, although their $\mathrm{TI}, \mathrm{Hg}$, and $\mathrm{Co}$ enrichment is rather large. Nevertheless, these minerals does not seem to be an important environmental issue as compared to similar accumulations known from the vicinity of acid mine drainage.

Acknowledgements. This work was financially supported by the Ministry of Science and Higher Education for the statutory objectives of the Institute of Geological Sciences PAS for the year 2015. The author would like to thank M. Krajcarz, Ph.D., for providing the samples; to R. Orłowski, Ph.D., for assistance by EDS analyses; and to Mr. A. Abdel-Rahman - for kindly encouraging to write this paper. 


\section{REFERENCES}

Ackermann, S., Lazic, B., Armbruster, T., Doyle, S., Grevel, K.-D., Majzlan, J., 2009. Thermodynamic and crystallographic properties of kornelite $\left[\mathrm{Fe}_{2}\left(\mathrm{SO}_{4}\right)_{3} \cdot \sim 7.75 \mathrm{H}_{2} \mathrm{O}\right]$ and paracoquimbite $\left[\mathrm{Fe}_{2}\left(\mathrm{SO}_{4}\right)_{3} \cdot 9 \mathrm{H}_{2} \mathrm{O}\right]$. American Mineralogist, 94: 1620-1628.

Anderson, J.L., Peterson, R.C., Swainson, I., 2009. The atomic structure and hydrogen bonding of deuterated melanterite, $\mathrm{FeSO}_{4} \cdot 7 \mathrm{D}_{2} \mathrm{O}$. The Canadian Mineralogist, 45: 457-469.

Anthony, J.W., Bideaux, R.A., Bladh, K.W., Nichols, M.C. eds., 2003. Handbook of Mineralogy (vol. 5),, Mineralogical Society of America, Chantilly, VA 20151-1110, USA; http: /www.handbookofmineralogy.org

Bandy, M.C., 1938. Mineralogy of three sulphate deposits of Northern Chile. Journal of the Mineralogical Society of America, 23: $670-760$.

Baur, W.H., 1964. On the crystal chemistry of salt hydrates. III. The Determination of the Crystal Structure of $\mathrm{FeSO}_{4} \cdot 7 \mathrm{H}_{2} \mathrm{O}$ (Melanterite). Acta Crystallographica, 17: 1167-1174.

Baur, W.H., Rolin, J.L., 1972. Salt hydrates. IX. The comparison of the crystal structure of magnesium sulphate pentahydrate with copper sulphate pentahydrate and magnesium chromate pentahydrate. Acta Crystallographica, 28: 1448-1455.

Bayliss, P., Atencio, D., 1985. X-ray powder-diffraction data and cell parameters for copiapite-group minerals. Zeitschrift für Kristallographie, 135: 34-55.

Berry, L.G., 1947. Composition and optics of copiapite. University of Toronto Studies, Geological Series, 51: 21-34.

Bielowicz, B., Misiak, J., 2016. Siarczki w pokładach węgla kamiennego warstw orzeskich s.s. serii mułowcowej (westfal B) we wschodniej części GZW (in Polish). Gospodarka Surowcami Mineralnymi - Mineral Resources Management, 32: 23-38.

Blass, G., Strehler, H., 1993. Mineralbildungen in einer durch Selbstentzündung brennenden Bergehalde des Aachener Steinkohlenreviers. Mineralien-Welt, 4: 35-42.

Bobos, I., Durães, N., Noronha, F., 2006. Mineralogy and geochemistry of mill tailings impoundments from Algares (Aljustrel), Portugal: implications for acid sulfate mine waters formation. Journal of Geochemical Exploration, 88: 1-5.

Buckby, T., Black, S., Coleman, M.L., Hodson, M.E., 2003. Fe-sulphate-rich evaporative mineral precipitates from the Río Tinto, southwest Spain. Mineralogical Magazine, 67: 263-278.

Chipera, S.J., Vaniman, D.T., 2007. Experimental stability of magnesium sulfate hydrates that may be present on Mars. Geochimica et Cosmochimica Acta, 71: 241-250.

Cooper, J.F., Jr., Dunning, G.E., Hadley, T.A., Moller, W.P., Reynolds, R.E., 2005. The sulfur hole, Calico Disctrict, San Bernardino County, California. Axis, 1: 1-18.

Čech, F., 1979. Rostite, a new name for orthorhombic $\mathrm{Al}\left(\mathrm{SO}_{4}\right)(\mathrm{OH})$ $5 \mathrm{H}_{2} \mathrm{O}$. Neues Jahrbuch für Mineralogie - Monatshefte: 193-194.

Dai, S., Liu, J., Ward, C.R., Hower, J.C., Xie, P., Jiang, Y., Hood, M.M., O’Keefe, J.M.K., Song, H., 2015. Petrological, geochemical, and mineralogical compositions of the low-Ge coals from the Shengli Coalfield, China: a comparative study with Ge-rich coals and a formation model forcoal-hosted Ge ore deposit. Ore Geology Reviews, 71: 318-349.

Dai, S., Wang, X., Zhao, L., 2017. Mineral Matter and Trace Elements in Coal, Special Issue, 1st ed. MDPI Books, Basel, Switzerland.

Demartin F., Castellaro, C., Gramaccioli, C.M., Campostrini, I., 2010. Aluminium-for-iron substitution, hydrogen bonding, and a novel structure-type in coquimbite-like minerals. The Canadian Mineralogist, 48: 323-333.

Dill, H.G., Pöllmann, H., Bosecker, K., Hahn, L., Mwiya, S., 2002. Supergene mineralization in mining residues of the Matchless cupreous pyrite deposit (Namibia) - a clue to origin of modern and fossil duricrusts in semiarid climates. Journal of Geochemical Exploration, 75: 43-70.

Dokoupilová, P., Sracek, O., Losos, Z., 2007. Geochemical behaviour of and mineralogical transformations during spontane- ous combustion of a coal waste pile in Oslavany, Czech Republic. Mineralogical Magazine, 71: 443-460.

ELARD, 2017. Updated master plan for the closure and rehabilitation of uncontrolled dumpsites thorough the country of Lebanon. Earth Link and Advanced Resources Development, Vol. A, United Nations Development Programme (UNDP) and the Ministry of Environment, http://www.lb.undp.org/content/lebanon/en/home/library/environment_energy/MASTER-PLAN-FOR-THE-CLOSURE-AND-REHABBILITATION.html

Fanfari, L., Nunzi, A., Zanzari, P.F., Zanzari, A.R., 1973. The copiapite problem: the crystal structure of a ferrian copiapite. American Mineralogist, 58: 314-322.

Fang, J.H., Robinson, P.D., 1970. Crystal structures and mineral chemistry of hydrated ferric sulfates. I. The crystal structure of coquimbite. American Mineralogist, 55: 1534-1540.

Fang, J.H., Robinson, P.D., 1976. Alunogen, $\mathrm{Al}_{2}\left(\mathrm{H}_{2} \mathrm{O}\right)_{12}\left(\mathrm{SO}_{4}\right)_{3}$ $5 \mathrm{H}_{2} \mathrm{O}$ : its atomic arrangement and water content. American Mineralogist, 61: 311-317.

Fernández-Remolar, D.C., Morris, R.V., Gruener, J.E., Amils, R., Knoll, A.H., 2005. The Río Tinto Basin, Spain: mineralogy, sedimentary geobiology, and implications for interpretation of outcrop rocks at Meridiani Planum, Mars. Earth and Planetary Science Letters, 240: 149-167.

Filippidis, A., Georgakopoulos, A., Kassoli-Fournaraki, A., 1996. Mineralogical components of some thermally decomposed lignite and lignite ash from the Ptolemais basin, Greece. International Journal of Coal Geology, 30: 303-314.

Fitzpatrick, R., Shand, P., Raven, M., McClure, S., 2010. Occurrence and environmental significance of sideronatrite and other mineral precipitates in Acid Sulfate Soils. 19th World Congress of Soil Science, Soil Solutions for a Changing World, 1-6 August 2010, Brisbane, Australia: 80-83.

Gluskoter, H.J., 1977. Inorganic sulfur in coal. Energy Sources, 3: 125-131.

Goldschmidt, V.M., 1937. The principles of distribution of chemical elements in minerals and rocks. 7th Hugo Müller Lecture; Journal of the Chemical Society (Resumed): 655-673.

Gornitz, V., 2004. Minerals as keys to ancient climates. Mineral News, 20: 9-13.

Gornitz, V., 2005. Mapping the minerals on Mars. Mineral News, 21: $1-11$.

Hammarstrom, J.M., Seal II, R.R., Meier, A.L., Kornfeld, J.M., 2005. Secondary sulphate minerals associated with acid mine drainage in the eastern US: recycling of metals and acidity in surficial environments. Chemical Geology, 215: 407-431.

Hudson-Edwards, K.A., Schell, C., Macklin, M.G., 1999. Mineralogy and geochemistry of alluvium contaminated by metal mining in the Rio Tinto, southwest Spain. Applied Geochemistry, 14: 1015-1030.

Jamieson, H.E., Robinson, C., Alpers, C.N., McCleskey, R.B., Nordstrom, D.K., Peterson, R.C., 2005. Major and trace element composition of copiapite-group minerals and coexisting water from the Richmond mine, Iron Mountain, California. Chemical Geology, 215: 387-405.

Joeckel, R.M., Ang Clement, B.J.A., Van Fleet Bates, L.R., 2005. Sulfate-mineral crusts from pyrite weathering and acid rock drainage in the Dakota Formation and Graneros Shale, Jefferson County, Nebraska. Chemical Geology, 541: 69.

Kampf, A.R., Mills, S.J., Housley, R.M., Williams, P.A., Dini, M., 2012. Alcaparrosaite, $\mathrm{K}_{3} \mathrm{Ti}^{4+} \mathrm{Fe}^{3+}\left(\mathrm{SO}_{4}\right)_{4} \mathrm{O}(\mathrm{OH})_{2}$, a new hydrophobic $\mathrm{Ti}^{4+}$ sulfate from Alcaparrosa, Chile. Mineralogical Magazine, 76: 851-861.

Keith, D.C., Runnels, D.D., Esposito, K.L., Chermak, L.A., Levy, D.B., Hannula, S.R., Watts, M., Hall, L., 2001. Geochemical models of the impact of acid groundwater and evaporative sulfate salts on Boulder Creek at Iron Mountain, California. Applied Geochemistry, 16: 947-961.

Ketris, M.P., Yudovich, Ya.E., 2009. Estimations of clarkes for carbonaceous biolithes: World averages for trace element contents 
in black shales and coals. International Journal of Coal Geology, 78: $135-148$.

Kostova, I., Marinov, S., Stefanova, M., Markova, K., Stamenova, V., 2005. The distribution of sulphur forms in high-S coals of the Maritza West Basin, Bulgaria. Bulletin of Geosciences, 80: 23-32.

Kruszewski, Ł., 2006. Oldhamite-periclase-portlandite-fluorite assemblage and coexisting minerals from burnt dump in Siemianowice Sląskie - Dąbrówka Wielka area (Upper Silesia, Poland) - preliminary report. Mineralogia Polonica - Special Papers, 28: 118-120.

Kruszewski, $七$., 2013. Supergene sulphate minerals from the burning coal mining dumps in the Upper Silesian Coal Basin, South Poland. International Journal of Coal Geology, 105: 91-109.

Kruszewski, Ł., Fabiańska, M.J., Ciesielczuk, J., Segit, T., Orłowski, R., Motyliński, R., Moszumańska, I., Kusy, D. 2018a. First multi-tool exploration of a gas-condensate-pyrolysate system from the environment of burning coal mine heaps: an in situ FTIR and laboratory GC and PXRD study based on Upper Silesian materials. Science of the Total Environment, 640-641: 1044-1071.

Kruszewski, Ł., Gatel, P., Thiéry, V., Moszumańska, I., Kusy, D., 2018b. Crystallochemical behavior of slag minerals and the occurrence of potentially new mineral species from Lapanouse-de-Sévérac, France. Coal and Peat Fires, 5: 241-300.

Lafuente, B., Downs, R.T., Yang, H., Stone, N., 2015. The power of databases - the RRUFF project, In: Highlights in Mineralogical Crystallography (eds. T. Armbruster and D.M. Danisi): 1-30. W. De Gruyter.

Lebanon Ministry of Tourism, 2011. destinationlebanon.gov.lb/content/uploads/DiscoverLebanonDetail/140429124241884 BOOK_SOUTH.pdf

Lipiarski, I., Muszyński, M., Wyszomirski, P., 2004. Alunites in the Red Beds of the „Marcel” coal mine, Upper Silesian Coal Basin, Poland. Mineralogia Polonica, 35: 3-18.

Liu, J., Ward, C.R., Graham, I.T., French, D., Dai, S., Song, X., 2018. Modes of occurrence of non-mineral inorganic elements in lignites from the Mile Basin, Yunnan Province, China. Fuel, 222: $146-155$.

Lovas, G.A., 1986. Structural strudy of halotrichite from Recsk (Mátra Mts., N-Hungary) (in Hungarian with English summary). Acta Geologica Hungarica, 29: 389-398.

Lutz Ehrlich, H., Newman, D.K., 2009. Geomicrobiology. 5th Ed., CRC Press, Taylor Francis Group, Boca Raton, FL, USA.

Majzlan, J., Michalik, R., 2007. The crystal structures, solid solutions and infrared spectra of copiapite-group minerals. Mineralogical Magazine, 71: 553-559

Majzlan, J., Alpers, C.N., Koch, Bender Koch, C., McCleskey, R.B., Myneni, S.C.B., Neil, J.M., 2011. Vibrational, X-ray absorption, and Mössbauer spectra of sulfate minerals from the weathered massive sulfide deposit at Iron Mountain, California. Chemical Geology, 284: 296-305.

Martin, R., Rodgers, K.A., Browne, P.R.L., 1999. The nature and significance of sulphate-rich, aluminous efflorescences from the Te Kopia geothermal field, Taupo volcanic zone, New Zealand. Mineralogical Magazine, 63: 413-419.

Masalehdani, M.N.-N., Mees, F., Dubois, M., Coquinot, Y., Potdevin, J.-L., Fialin, M., Blanc-Valleron, M.-M., 2009. Condensate minerals from a burning coal-waste heap in Avion, northern France. The Canadian Mineralogist, 47: 573-591.

Menchetti, S., Sabelli, C., 1974. Alunogen. Its structure and twinning. Tschermaks Mineralogische und Petrographische Mitteilungen, 21: 164-178.

Mereiter, K., 2013. Redetermination of tamarugite, $\mathrm{NaAl}\left(\mathrm{SO}_{4}\right)_{2}$ $6 \mathrm{H}_{2} \mathrm{O}$. Acta Crystallographica, E69: i63-i64.

Montano, P.A., 1981. Characterization of iron-bearing minerals in coal. Advances in Chemistry, Ch. 22, 192: 337-361.

Miura, H., Niida, K., Hirama, T., 1994. Mikasaite, $\left(\mathrm{Fe}^{3+}, \mathrm{Al}\right)_{2}\left(\mathrm{SO}_{4}\right)_{3}$, a new ferric sulphate mineral from Mikasa County, Hokkaido, Japan. Mineralogical Magazine, 58: 649-653.
Murray, J., Kirschbaum, A., Dold, B., Mendes Guimaraes, E., Pannunzio Miner, E., 2014. Jarosite versus soluble iron-sulfate formation and their role in acid mine drainage formation at the Pan de Azúcar Mine Tailings (Zn-Pb-Ag), NW Argentina. Minerals, 4: 477-502.

Nanhas, C., 2012. Jezzine fifth highest waterfall in the world. National News Agency (NNA) News; nna-leb.gov.lb/en/show-report/82/Jezzine-fifth-highest-waterfall-in-the-world

O'Connor, V.A., 2005. Comparative crystal chemistry of hydrous iron sulfates from different terrestrial environments. B.A. thesis, Department of Geology of Smith College.

Palache, C., Berman, H., Frondel, C., 1951. Dana's system of mineralogy, 7th ed., 2, 495-496.

Parker, R.L., 1967. Composition of the Earth's crust. Data of Geochemistry (ed. M. Fleischer). Geological Survey Professional Paper 440-D, United States Government Printing Office, Washington, D1-D19

Parzentny, H.R., Lewińska-Preis, R., 2006. The role of sulphide and carbonate minerals in the concentration of chalcophile elements in the bituminous coal seams of a paralic series (Upper Carboniferous) in the Upper Silesian Coal Basin (USCB), Poland. Chemie der Erde, 66: 227-247.

Pecharsky, V.K., Zavalij, P.Y., 2003. Fundamentals of powder diffraction and structural characterization of materials. Springer Science+Business Media, Inc., New York, USA

Pekov, I.V., Siidra, O.I., Chukanov, N.V., Yapaskurt, V.O., Belakovskiy, D.I., Turchkova, a.G., Möhn, G., 2016. Calamaite, IMA 2016-036. CNMNC Newsletter, 33, October 2016: 1136; Mineralogical Magazine, 80: 1135-1144.

Peterson, R.C., 2003. The relationship between Cu content and distortion in the atomic structure of melanterite from the Richmond mine, Iron Mountain, California. The Canadian Mineralogist, 41: 937-949.

Quartieri, S., Triscari, M., Viani, A., 2000. Crystal structure of the hydrated sulphate pickeringite $\left(\mathrm{MgAl}_{2}\left(\mathrm{SO}_{4}\right)_{4} \cdot 22 \mathrm{H}_{2} \mathrm{O}\right.$ : X-ray powder diffraction study. European Journal of Mineralogy, 12: 1131-1138.

Ramusino, C.C., Giuseppetti, G., 1973. Ritrovamento di un materiale naturale riferible al composto sintético: $6 \mathrm{Fe}_{2}\left(\mathrm{SO}_{4}\right)_{3}$. $\mathrm{Fe}_{2} \mathrm{O}_{3} . \mathrm{xH} 2 \mathrm{O}$ nelle miniere d'oro di Challant - St. Anselme (Valle d'Ayas) (in Italian). Natura Rivisita di Scienze Naturali, 64: 45-460

Rice, M.S., Bell III, J.F., Cloutis, E.A., Wang, A., Ruff, S.W., Craig, M.A., Bailey, D.T., Johnson, J.R., de Souza, P.A., Farrand, W.H., 2010. Silica-rich deposits and hydrated minerals at Gutsev Crater, Mars: Vis-NIR spectral characterization and regional mapping. Icarus, 205: 375-395.

Robinson, P.D., Fang, J.H., 1969. Crystal structure and mineral chemistry of double-salt hydrates: I. Direct determination of crystal structure of tamarugite. American Mineralogist, 54: 19-30.

Robinson, P.D., Fang, J.H., 1971. Crystal structures and mineral chemistry of hydrated ferric sulfates. II. The crystal structure of paracoquimbite. American Mineralogist, 56: 1567-572.

Rodgers, K.A., Hamlin, K.A., Borwne, P.R.L., Campbell, K.A., Martin, R., 2000. The steam condensate alteration mineralogy of Ruatapu cave, Orakei Korako geothermal field, Taupo Vocanic Zone, New Zealand. Mineralogical Magazine, 64: 125-142.

Romero, A., González, I., Galán, E., 2016. The role of efflorescent sulfates in the storage of trace elements in stream waters polluted by acid mine-drainage: the case of Peña del Hierro, Southwestern Spain. The Canadian Mineralogist, 44: 1431-1446.

Rosse, H., 1883. Ueber einige in Südamerika vorkommende Eisenoxydsalze. Annalen der Physik und Chemie, 72, J.C. Poggendorf, Berlin: 309-319.

Segnit, E.R., 1976. Tamarugite from Anglesea, Victoria, Australia. Mineralogical Magazine, 40: 642-644.

Sgavetti, M., Pompillo, L., Roveri, M., Manzi, V., Valentino, G.M., Lugli, S., Carli, C., Amici, S., Marchese, F., Lacava, T., 2009. Two geologic systems providing terrestrial analogues for the ex- 
ploration of sulfate deposits on Mars: initial spectral characterization. Planetary and Space Science, 57: 614-627.

Skarpelis, N., Argyraki, A., 2009. The geology and origin of supergene ores in Lavrion (Attica, Greece). Resource Geology, 59: 1-14.

Smith, G.I., Almond, H., Sawyer, D.L., Jr., 1958. Sassolite from the Kramer borate district, California. American Mineralogist, 43: 1074.

Smuda, J., Dold, B., Friese, K., Morgenstern, P., Glaesser, W., 2007. Mineralogical and geochemical study of element mobility at the sulfide-rich excelsior waste rock dump from the polymetallic $\mathrm{Zn}-\mathrm{Pb}-(\mathrm{Ag}-\mathrm{Bi}-\mathrm{Cu})$ deposits, Cerro de Pasco, Peru. Journal of Geochemical Exploration, 92: 97-110.

Stracher, G.B., Prakash, A., Schroeder, P., McCormack, J., Zhang, X., Van Dijk, P., Blake, D., 2005. New mineral occurrences and mineralization processes: Wuda coal-fire gas vents of Inner Mongolia. American Mineralogist, 90: 1729-1739.

Susilawati, R., Ward, C.R., 2006. Metamorphism of mineral matter in coal from the Bukit Asam deposit, south Sumatra, Indonesia. International Journal of Coal Geology, 68: 171-195.

Süsse, P., 1972. Crystal structure and hydrogen bonding of copiapite. Zeitschrift für Kristallographie, 135: 34-55.

Triantafyllidis, S., Skarpelis, N., 2006. Mineral formation in acid pit lake from a high-sulfidation ore deposit: Kirki, NE Greece. Journal of Geochemical Exploration, 88: 68-71.

Velasco, F., Alvaro, A., Suarez, S., Herrero, J.-M., Yusta, I., 2005. Mapping Fe-bearing hydrated sulphate minerals with short wave infrared (SWIR) spectral analysis at San Miguel mine environment, Iberian Pyrite Belt (SW Spain). Journal of Geochemical Exploration, 87: 45-72.

Walley, C.D., 1997. The lithostratigraphy of Lebanon: a review. Lebanese Science Bulletin, 10: 81-108.

Ward, C.R., 2002. Analysis and significance of mineral matter in coal seams. International Journal of Coal Geology, 50: $135-138$.
Ward, C.R., 2016. Analysis, origin and significance of mineral matter incoal: an updated review. International Journal of Coal Geology, 165: 1-27.

Welch, S.A., Christy, A.G., Isaacson, L., Kirste, D., 2009. Mineralogical control of the rare earth elements in acid sulphate soils. Geochimica et Cosmochimica Acta, 73: 44-64.

Wildner, M., Giester, G., 1991. The crystal structure of kieserite-type compounds. I. Crystal structures of $\mathrm{Me}(\mathrm{II}) \mathrm{SO}_{4}$. - $\mathrm{H}_{2} \mathrm{O}(\mathrm{Me}=\mathrm{Mn}, \mathrm{Fe}, \mathrm{Co}, \mathrm{Ni}, \mathrm{Zn})$. Neues Jahrbuch für Mineralogie Monatshefte: 296-306.

Wisotzky, F., Obermann, P., 2001. Acid mine groundwater in lignite overburden dumps and its prevention - the Rhineland lignite mining area (Germany). Ecological Engineering, 17: 115-123.

Witzke, T., 1996. Die Minerale der brennenden Halde der Steinkohlengrube "Deutschland-schacht" in Oelsnitz bei Zwickau. Aufschluss, 47: 41-48.

Yalovik, L., Tatarinov, A., Danilova, E., Doroshkevich, S., 2016. Bio-inert Dome and Columnar Structures of Mud Microvolcanism in Baikal Rift Zone. International Journal of Advanced Research in Science, Engineering and Technology, 3: 2589-2600.

Yang, Z., Giester, G., 2018. Structure refinements of coquimbite and paracoquimbite from the Hongshan $\mathrm{Cu}-\mathrm{Au}$ deposit, NW China. European Journal of Mineralogy, 30: 849-858.

Young, B., Nancarrow, P.H.A., 1988. Rozenite and other sulphate minerals from the Cumbrian coalfield. Mineralogical Magazine, 52: $551-553$.

Zodrow, E.L., 1980. Hydrated sulfates from Sydney Coalfield, Cape Breton Island, Nova Scotia, Canada: the copiapite group. American Mineralogist, 65: 961-967.

Zielinski, R.A., Otton, J.K., Johnson, C.A., 2001. Sources of salinity near a coal mine spoil pile, north-central Colorado. Journal of Environmental Quality, 30: 1237-1248.

Zimbelman, D.R., Rye, R.O., Breit, G.N., 2005. Origin of secondary sulfate minerals on active andesitic stratovolcanoes. Chemical Geology, 215: 37-60. 